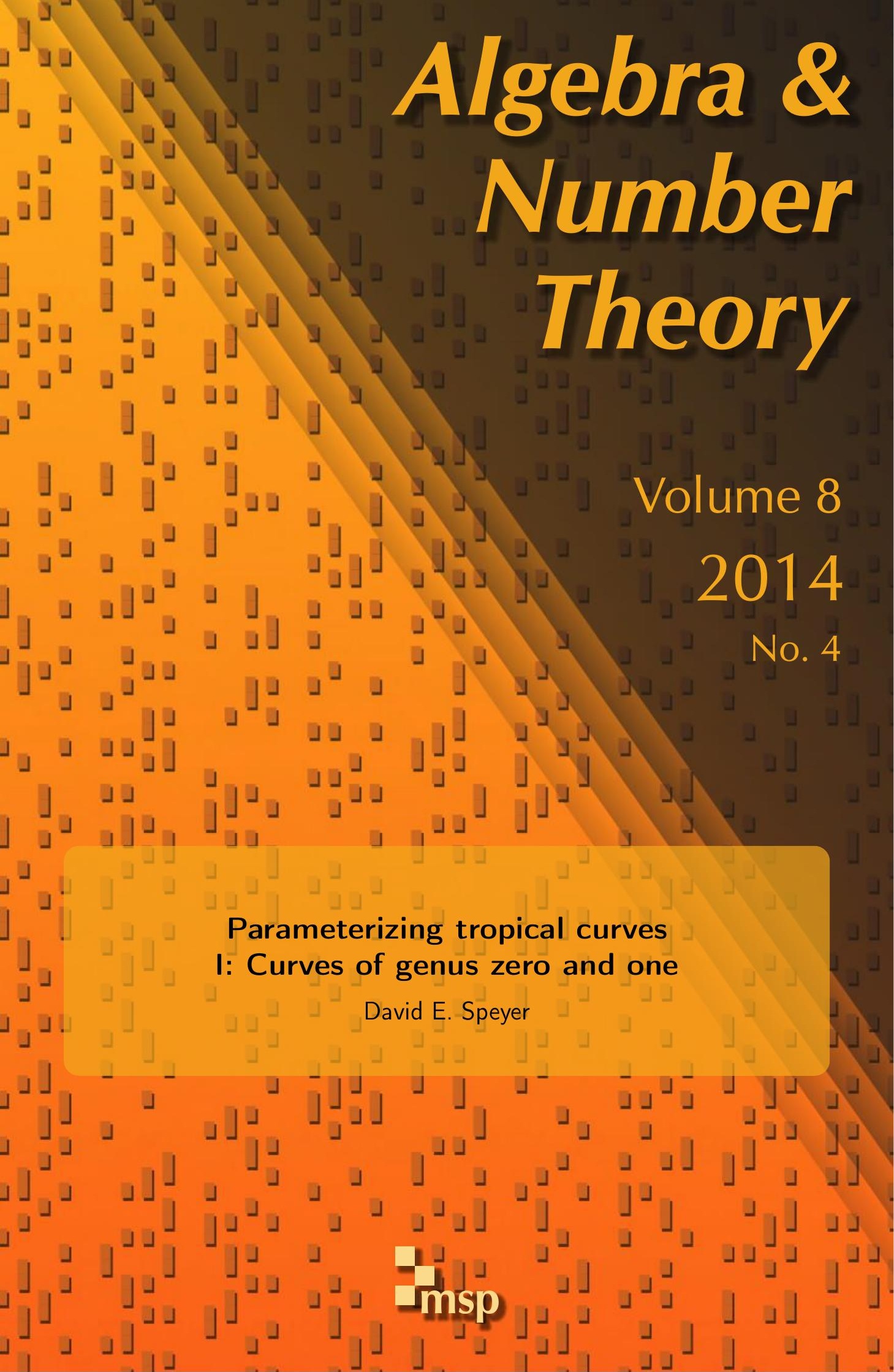




\title{
Parameterizing tropical curves I: Curves of genus zero and one
}

\author{
David E. Speyer
}

In tropical geometry, given a curve in a toric variety, one defines a corresponding graph embedded in Euclidean space. We study the problem of reversing this process for curves of genus zero and one. Our methods focus on describing curves by parameterizations, not by their defining equations; we give parameterizations by rational functions in the genus-zero case and by nonarchimedean elliptic functions in the genus-one case. For genus-zero curves, those graphs which can be lifted can be characterized in a completely combinatorial manner. For genus-one curves, we show that certain conditions identified by Mikhalkin are sufficient and we also identify a new necessary condition.

1. Curves in toric varieties 965

2. Basic tropical background 967

3. Statement of results 971

4. The Bruhat-Tits tree 974

5. Lemmas on zero-tension curves 976

6. Tropical curves of genus zero 977

7. Tropical curves of genus one 981

8. Superabundant curves 991

9. The necessity of well-spacedness and the $j$-invariant 994

Acknowledgements 996

References $\quad 996$

In the past ten years, a group of mathematicians, led by Grigory Mikhalkin, have pioneered a new method for studying curves in toric varieties. According to this perspective, one considers curves defined over a field with a nonarchimedean valuation. Using this valuation and an embedding of a curve $C$ into an (algebraic) torus, one constructs a graph embedded in a real vector space. This graph is known as the tropicalization of the curve. From the tropicalization of $C$, one tries to read off information about the degree and genus of the original curve $C$, and its intersections

MSC2010: 14T05.

Keywords: tropical geometry, curves, nonarchimedean, Tate curve. 
with other subvarieties of the torus. In this introduction, we will write $C$ for a curve embedded in a torus $T \cong\left(\mathbb{K}^{*}\right)^{n}$ and we will write $\Gamma \subset \mathbb{R}^{n}$ for the tropicalization of $C$.

In order to use these tropical methods, we need to know which graphs are tropicalizations of curves. We will refer to a graph which actually is the tropicalization of a curve as a tropical curve. There are certain basic combinatorial conditions which hold for any tropical curve. The first, the zero-tension condition, is a description of the possible local structures of a tropical curve around a given vertex (see the beginning of Section 3). We can assign to $C$ a multiset of lattice vectors, which we will call the degree of $C$, from which we can determine the homology class represented by the closure of $C$ when this closure is taken in a suitable toric compactification of $T$. The second combinatorial condition is that the directions of the unbounded rays of $\Gamma$ are given by the degree of $C$ (Section 1). Thirdly, we can show that, modulo some technical conditions, the genus of $C$ is greater than or equal to the first Betti number of $\Gamma$ (see Theorem 3.1). We will define a zero-tension curve of genus $g$ and degree $\delta$ to be a graph which has first Betti number $g$ and obeys the obvious conditions to be the tropicalization of a degree- $\delta$ curve.

We attack the reverse problem: given a zero-tension curve of genus $g$ and degree $\delta$, when does it come from an actual curve of genus $g$ and degree $\delta$ ? The main contribution of this paper is to show that methods of nonarchimedean analysis can be used to construct algebraic curves with a given tropicalization. In this paper, we will consider this question for genus-zero and genus-one curves. In the sequel, we will describe the corresponding results for higher-genus curves, where we will need to use Mumford's uniformization results. A second achievement of this paper is to describe a condition - that of being well spaced - which permits us to conclude that some "obstructed" curves of genus one can nonetheless be lifted.

I want to state clearly that there is a major difficulty in directly using these results for enumerative purposes involving curves of positive genus. If $C$ is a curve of genus $g$, than the tropicalization of $C$ is a zero-tension curve with first Betti number less than or equal to $g$. Therefore, if we want to count genus- $g$ curves obeying some conditions, we should look at all zero-tension curves of genus less than or equal to $g$, and determine which of them lift to actual genus- $g$ curves obeying the condition (and in how many ways the lifting can be done). However, we have almost no results restricting the capability to lift a tropical curve whose first Betti number is strictly less than $g$ to an actual curve of genus $g$. When studying curves in toric surfaces, one can use basic dimension-counting arguments to show that there are no such contributions, but this cannot be done for curves in higher-dimensional toric varieties. I expect, therefore, that the primary use of these results will not be to prove exact combinatorial formulas, but rather to provide existence results or lower bounds. 
The idea of studying curves via tropical varieties was proposed by Kontsevich and pioneered by Mikhalkin [2005; 2006]. Mikhalkin has proven our main theorems, in any genus, in the case of curves in toric surfaces. A purely algebraic proof was given by Shustin and Tyomkin [2006]. Since Mikhalkin's work, there has been a great deal of research extending his results to more sophisticated enumerative problems concerning curves in toric surfaces. There has been far less work on curves in higher-dimensional toric varieties. The most important result in higher dimensions is that of Nishinou and Siebert [2006], who use log geometry to analyze the case of genus-zero curves and recover essentially all of our results in that case. Finally, we should note that H. Markwig and her collaborators, especially Gathmann, have done major work building the tropical analogue of the moduli spaces of curves and of stable maps and studying it from a combinatorial perspective; see [Gathmann et al. 2009] and the works cited therein. Among their results is reestablishing the validity of the tropical enumeration of curves in $\mathbb{P}^{2}$ by showing it matches the Caporaso-Harris formula. The moduli space of tropical genus-zero curves was previously described by Mikhalkin [2007].

Newer work. Since this paper was first prepared, several additional relevant papers have appeared. Of particular relevance are the papers of Baker, Payne and Rabinoff [Baker et al. 2012], Helm and Katz [2012], Katz [2012], and Nishinou [2010]. The first is an exhaustive discussion of parametrization of nonarchimedean curves using the language of Berkovich's analytic spaces. In terms of results, this paper goes beyond [Baker et al. 2012] in that we prove that our combinatorially necessary conditions are sufficient to realize tropical curves. In terms of exposition, I hope that the use of the Bruhat-Tits tree rather than Berkovich spaces removes one level of technical requirement.

Helm and Katz relate the lengths of the cycles in the tropical curve to the monodromy action on the cohomology of the general fiber, generalizing the relationship shown in this paper between the length of the cycle in genus one and the valuation of the $j$-invariant. Katz defines several obstructions to tropical lifting which generalize the well-spacedness condition from this paper.

Nishinou provides an alternate proof that ordinary tropical curves are realizable (in all genuses), using log structures, and provides additional analysis of the superabundant case.

\section{Curves in toric varieties}

In this section, we will describe how to assign a degree to a curve given with a map to an (algebraic) torus. Throughout this paper, we will write $N$ for the lattice of one-parameter subgroups of the torus and $M$ for the character lattice. We will call 
the dimension of the torus $n$. We write $\mathbb{T}$ for the torus, or $\mathbb{T}(\mathbb{K}, N)$ when we want to specify the ground field $\mathbb{K}$ and the lattice $N$.

Let $\Sigma$ be a complete rational fan in $\mathbb{Q} \otimes N$ and let $\mathrm{X}(\Sigma)$ be the associated toric variety over an algebraically closed field $\mathbb{K}$. (See [Fulton 1993] for background on toric varieties.) The open torus in $\mathrm{X}(\Sigma)$ is $\operatorname{Hom}\left(N, \mathbb{K}^{*}\right)$. For each ray (onedimensional cone) of $\Sigma$, there is a unique minimal element of $N$ on this ray; if we identify $N$ with $\mathbb{Z}^{n}$, then the element in question is the unique point of the ray whose coordinates are integers with no common factor. Let $\rho_{1}, \ldots, \rho_{p}$ be the set of minimal vectors of the rays of $\Sigma$. The following is a special case of Theorem 3.1 of [Fulton and Sturmfels 1997].

Proposition 1.1. With the notation above, the Chow group $A^{n-1}$ is given by

$$
A^{n-1}(\mathrm{X}(\Sigma)) \cong\left\{\left(d_{1}, d_{2}, \ldots, d_{p}\right) \in \mathbb{Z}^{p}: \sum_{i=1}^{p} d_{i} \rho_{i}=0\right\} .
$$

This is a subgroup of $H^{2 n-2}(\mathrm{X}(\Sigma), \mathbb{Z})$, and equals $H^{2 n-2}(\mathrm{X}(\Sigma), \mathbb{Z})$ if $\mathrm{X}(\Sigma)$ is smooth.

Let $\bar{C}$ be a smooth, complete algebraic curve and let $\phi: \bar{C} \rightarrow \mathrm{X}(\Sigma)$ be a map from $\bar{C}$ into $\mathrm{X}(\Sigma)$. Let $C$ denote $\phi^{-1}(\mathbb{T}) \subset \bar{C}$, the part of $\bar{C}$ which is mapped to the big torus. Let us say that $(\phi, \bar{C})$ is torically transverse if $C$ is nonempty and $\phi(\bar{C})$ is disjoint from the toric strata of codimension two and higher. (This is a specialization of the definition of torically transverse in [Nishinou and Siebert 2006].)

For each ray $\mathbb{Q}_{\geq 0} \rho_{i}$ of $\Sigma$, let $Y_{i}$ be the codimension-one stratum of $\mathrm{X}(\Sigma)$ associated to $\rho_{i}$. Let $d_{i}$ be the length of $\phi^{*}\left(\mathscr{O}_{Y_{i}}\right)$, in other words, the number of points in $\phi^{-1}\left(Y_{i}\right)$ counted with multiplicity. Then $\left(d_{1}, \ldots, d_{N}\right)$ satisfies ${ }^{1} \sum d_{i} \rho_{i}=0$ and hence corresponds to a class in $A^{n-1}(\mathrm{X}(\Sigma))$. Capping with the fundamental class gives the class in $A_{1}(\mathrm{X}(\Sigma))$, and hence in $H_{2}(\mathrm{X}(\Sigma))$, corresponding to $C$. Thus, if we want to study torically transverse curves representing a particular class in $H_{2}$, we may begin by finding the possible preimages of this class in $A^{n-1}$ and studying curves of that degree.

Continue to assume that $(\phi, \bar{C})$ is torically transverse. Let $x \in \bar{C} \backslash C$. We now describe how to determine which ray of $\Sigma$ corresponds to $x$, and with what multiplicity, solely by examining the map $C \rightarrow \mathbb{T}$. Namely, $M$ is the lattice of characters of the torus, so each element $\lambda$ of $M$ can be restricted to a function $\chi^{\lambda}$ on $C$. Let $\sigma_{x}$ be the map which sends $\lambda$ to the order of vanishing of $\chi^{\lambda}$ at $x$; the function $\sigma_{x}$ is in $N$. Write $\sigma_{x}$ as $d_{x} \rho_{x}$, where $d_{x}$ is a positive integer and $\rho_{x}$ is minimal. Then $\rho_{x}$ is the ray of $\Sigma$ corresponding to $x$, and $d_{x}$ is the multiplicity.

\footnotetext{
${ }^{1}$ In two paragraphs, we will see an alternate description of the $d_{i}$ that makes this clear.
} 
We now make a definition: let $C$ be a smooth algebraic curve and let $\phi: C \rightarrow \mathbb{T}$ be an algebraic map. Let $\bar{C}$ be the smooth complete curve compactifying $C$; we impose the condition that $\phi$ cannot be extended to any point of $\bar{C} \backslash C$. Let $x$ be any point of $\bar{C} \backslash C$. Define $\sigma_{x}, \rho_{x}$ and $d_{x}$ as before. Let $\rho_{1}, \ldots, \rho_{N}$ be the set of distinct values of $\rho_{x}$ as $x$ ranges over $\bar{C} \backslash C$. For $1 \leq i \leq N$, let $d_{i}=\sum_{\rho_{x}=\rho_{i}} d_{x}$ and set $\sigma_{i}=\sum_{\rho_{x}=\rho_{i}} \sigma_{x}=d_{i} \rho_{i}$. We define the set $\left\{\sigma_{1}, \ldots, \sigma_{N}\right\}$ to be the degree of $(\phi, C)$. Note that this is defined without any choice of toric compactification of $\mathbb{T}$. Note also that we have $\sum \sigma_{i}=0$, because any rational function has equally many zeroes and poles on $\bar{C}$.

We then have:

Proposition 1.2. Let $\Sigma$ be a complete fan with rays generated by $\rho_{1}, \ldots, \rho_{N}$ and let $\left(d_{1}, \ldots, d_{N}\right)$ be any class in $A^{n-1}(\mathrm{X}(\Sigma))$. There is a bijection between torically transverse curves $(\phi, \bar{C})$ which represent the given class and maps $C \rightarrow \mathbb{T}$ which are incapable of being extended to any larger compactification of $C$ and have degree $\left(d_{1} \rho_{1}, \ldots, d_{N} \rho_{N}\right)$; this bijection is given by restriction to the preimage of $\mathbb{T}$ in $\bar{C}$.

For this reason, we can reformulate questions about constructing torically transverse curves in toric varieties into questions about constructing curves of given degree in tori. Ordinarily, of course, one wishes to consider all curves in some toric variety, not only the torically transverse ones. In many applications, it can be shown by dimensional considerations that all of the curves of interest are torically transverse. Even when this is not true, it is true that, if we specify the cohomology class of a curve in $\mathrm{X}(\Sigma)$, then there are only finitely many possible degrees for curves realizing that cohomology class and not lying in the toric boundary - and those curves which do lie in the toric boundary are in the interiors of smaller toric varieties. For this reason, in this paper we will study problems where we specify the degree of a curve in the torus rather than specifying degrees in a toric compactification.

\section{Basic tropical background}

In this paper, we will study a great number of polyhedra. All of these will be rational polyhedra, meaning that they are defined by finitely many inequalities of the form $\left\{\left(x_{1}, \ldots, x_{n}\right): \sum a_{i} x_{i} \leq \lambda\right\}$, where $\left(a_{1}, \ldots, a_{n}\right)$ is an integer vector and $\lambda$ is a rational number. It will be most convenient to consider these as subsets of $\mathbb{Q}^{n}$ (for example, in Proposition 2.2). However, we want to be able to use topological language to talk about polyhedral complexes. We therefore adopt the following conventions: a polyhedron is a subset of $\mathbb{Q}^{n}$, defined by finitely many inequalities as above. When we refer to a point of a polyhedron, we mean a point of $\mathbb{Q}^{n}$. Nonetheless, when we describe a polyhedral complex using topological terms, such as "connected", "simply connected" and so forth, we will mean the properties of 
the closure of that complex in $\mathbb{R}^{n}$. Similar issues will arise concerning metrized graphs. We adopt the conventions that the edges of a metrized graph always have rational lengths and that the points of this graph, considered as a metric space, are the points which have rational distances from all of the vertices. Nevertheless, we will freely speak of graphs as connected, as mapping continuously from one to another, and so forth.

Let $\boldsymbol{O}$ be a complete discrete valuation ring with valuation $v: \boldsymbol{O} \rightarrow \mathbb{Z}_{\geq 0} \cup\{\infty\}$. We write $\boldsymbol{K}$ for the fraction field of $\boldsymbol{O}$ and $\kappa$ for the residue field. We always assume that $\kappa$ is algebraically closed. Let $\mathbb{K}$ be the algebraic closure of $\boldsymbol{K}$, let $v: \mathbb{K} \rightarrow \mathbb{Q} \cup\{\infty\}$ be the extension of $v$ to $\mathbb{K}$, let $\mathcal{O}$ be the ring $v^{-1}(\mathbb{Q} \geq 0 \cup\{\infty\})$, and let $\mathcal{M}$ and $\boldsymbol{M}$ be the maximal ideals of $\mathcal{O}$ and $\boldsymbol{O}$, respectively. Since $\kappa$ is algebraically closed, the residue field $\mathcal{O} / \mathcal{M}$ is also equal to $\kappa$. For $g \in \mathbb{O}$, write in $(g)$ for the class of $g$ in $\kappa$. We fix a group homomorphism $w \mapsto t^{w}$ from $\mathbb{Q} \rightarrow \mathbb{K}^{*}$ giving a section of $v: \mathbb{K}^{*} \rightarrow \mathbb{Q}$; this is always possible because $v$ is surjective and $\mathbb{K}^{*}$ is divisible. ${ }^{2}$ We will primarily consider the objects $\mathbb{K}, \mathcal{O}$ and $\mathcal{M}$ and only occasionally need to deal with $\boldsymbol{K}, \boldsymbol{O}$ and $\boldsymbol{M} .^{3}$

We will say that $\mathbb{K}$ is a power series field if we are given a section $\iota: \kappa \hookrightarrow \mathbb{O}$ such that in $(\iota(a))=a$. If $\mathbb{R}$ is a power series field, we will say that a polynomial $f \in \mathbb{K}[M]$ has constant coefficients if its coefficients lie in the image of $\kappa$ and that a variety $C \subset \mathbb{T}(\mathbb{K}, N)$ has constant coefficients if it is defined by polynomials with constant coefficients. The motivation for this terminology is that we can then think of $\boldsymbol{O}$ as the ring of formal power series in $t$, writing each element of $\boldsymbol{O}$ canonically as $\sum_{i} \iota\left(a_{i}\right) t^{i}$ for some sequence of elements $a_{i}$ in $\kappa$.

Let $M$ be a free abelian group of rank $n$ and let $N=\operatorname{Hom}(M, \mathbb{Z})$. We write $\langle$, for the pairing $M \times N \rightarrow \mathbb{Z}$. For $m \in M$, we will write $\chi^{m}$ for the corresponding element of the group ring $\mathbb{K}[M]$, so that $M$ can be written additively.

Let $f \in \mathbb{K}[M]$ and let $w \in \mathbb{Q} \otimes N$. We can write $f=\sum_{\lambda \in L} f_{\lambda} \chi^{\lambda}$, where $L$ is a finite subset of $M$ and each $f_{\lambda}$ is a nonzero element of $\mathbb{K}$. Let $L_{0}$ be the subset of $L$ on which the function $\lambda \mapsto v\left(f_{\lambda}\right)+\langle\lambda, w\rangle$ achieves its minimum. We define $\operatorname{in}_{w}(f)=\sum_{\lambda \in L_{0}} \operatorname{in}\left(t^{-v\left(f_{\lambda}\right)} f_{\lambda}\right) \chi^{\lambda}$, and we set $\operatorname{in}_{w}(0)=0$.

Suppose that $\mathbb{K}$ is a power series field and that $f \in \mathbb{K}[M]$ has constant coefficients, with $f=\sum_{\lambda \in L} \iota\left(f_{\lambda}\right) \chi^{\lambda}$. Then we can describe $\operatorname{in}_{w}(f)$ more simply as $\sum_{\lambda \in L_{0}} f_{\lambda} \chi^{\lambda}$; note that we sum over $L_{0}$, not over $L$. This is the definition that is used in Gröbner

\footnotetext{
${ }^{2}$ It is quite possible to do without this choice, and Sam Payne has advocated doing so. This is doubtless the most morally correct way of proceeding, but it introduces a great deal of notational baggage. In particular, we would be forced to replace tori with principal homogeneous spaces over tori in several places, and would thus no longer have explicit coordinates.

${ }^{3}$ Several earlier tropical works, including some of my own work, attempted to ignore $\boldsymbol{O}, \boldsymbol{K}$ and $\boldsymbol{M}$ entirely. The reader should still view these objects as being only technical crutches. However, I have become convinced that it is not worth trying to avoid them completely.
} 
theory, where the field $\mathbb{K}$ is left hidden in the background. Using this alternate definition, we can define $\operatorname{in}_{w}(f)$ for $f \in \kappa[M]$ and $w \in \mathbb{Q} \otimes N$, even without choosing a field $\mathbb{K}$ to use.

If $I$ is an ideal in $\mathbb{K}[M]$, let $\operatorname{in}_{w}(I)$ denote the ideal of $\kappa[M]$ generated by $\operatorname{in}_{w}(f)$ for $f \in I$. If $C$ is the closed subscheme of $\mathbb{T}(\mathbb{K}, N$ ) corresponding to $I$, then we write $\operatorname{in}_{w} C$ for the closed subscheme of $\mathbb{T}(\kappa, N)$ corresponding to $\operatorname{in}_{w} I$. The geometric meaning of $\operatorname{in}_{w} C$ is the following: let $t^{w}$ denote the element of $\mathbb{T}(\mathbb{K}, N)=\operatorname{Hom}\left(N, \mathbb{K}^{*}\right)$ described by $t^{w}(\lambda)=t^{\langle\lambda, w\rangle}$ for $\lambda \in N$. Consider the subvariety $t^{-w} \cdot C$ of $\mathbb{T}(\mathbb{K}, N)$, where $\cdot$ denotes the standard action of $\mathbb{T}(\mathbb{K}, N)$ on itself. Let $\overline{t^{-w} \cdot C}$ be the (Zariski) closure of $t^{-w} \cdot C$ in Spec O [M]. Then $\operatorname{in}_{w} C$ is the fiber of $\overline{t^{-w} C}$ over Spec $\kappa$. Moreover, suppose that $t^{w} \in \boldsymbol{K}$ and that $C$ is defined over $\boldsymbol{K}$, meaning that there is a subscheme $\boldsymbol{C}$ of $\mathbb{T}(\boldsymbol{K}, N)$ such that $C=\boldsymbol{C} \times \boldsymbol{K} \mathbb{K}$. (Note that we can always achieve these hypotheses by replacing $\boldsymbol{K}$ with a finite extension.) Then we may instead describe in ${ }_{w} C$ by taking the Zariski closure of $t^{-w} \boldsymbol{C}$ in $\operatorname{Spec} \boldsymbol{O}[M]$.

If $I$ is an ideal of $\kappa[M]$, rather than of $\mathbb{K}[M]$, and $w$ any point of $\mathbb{Q} \otimes N$, then we can define $\operatorname{in}_{w} I$ to be the ideal in $\kappa[M]$ generated by in $f$ for all $f \in I$, where $\operatorname{in}_{w} f$ is defined by the alternate definition two paragraphs above. While this is a slight abuse of notation, it should cause no confusion: one meaning of $\operatorname{in}_{w} I$ is defined for $I \subset \kappa[M]$ and the other for $I \subset \mathbb{K}[M]$ and the meanings are extremely closely related. Specifically, let $I$ be an ideal of $\kappa[\lambda]$, let $w \in \mathbb{Q} \otimes N$ and let $\mathbb{K}^{\prime}$ be any power series field with associated notation $\left(\mathbb{K}^{\prime}, v^{\prime}, \mathbb{O}^{\prime}, \mathcal{M}^{\prime}, \kappa^{\prime}\right)$. Suppose that $w \in \mathbb{Q} \otimes N$ and that $\kappa^{\prime}=\kappa$. Then $\operatorname{in}_{w} I=\operatorname{in}_{w}\left(\mathbb{K}^{\prime} \otimes_{\kappa^{\prime}} I\right)$.

The following lemma will be of frequent use:

Lemma 2.1. Let I be an ideal of $\mathbb{K}[M]$. Let $w$ and $v$ be elements of $\mathbb{Q} \otimes N$. Then, for any sufficiently small rational number $\epsilon$, we have $\mathrm{in}_{w+\epsilon v} I=\mathrm{in}_{v} \operatorname{in}_{w} I$.

Proof. This is proved in a less general context as Proposition 1.13 in [Sturmfels 1996]; the proof can be adapted to our setting.

We now define tropicalization.

Proposition 2.2. Let $C$ be a closed subscheme of $\mathbb{T}(\mathbb{K}, N)$ and let $I \subset \mathbb{K}[M]$ be the corresponding ideal. Let $w \in \mathbb{Q} \otimes N$. Then the following are equivalent:

(1) There is a point $x \in C(\mathbb{K})$ with $v(x)=w$.

(2) There is a valuation $\tilde{v}: \mathbb{K}[M] / I \rightarrow \mathbb{Q} \cup\{\infty\}$ extending $v: \mathbb{K} \rightarrow \mathbb{Q} \cup\{\infty\}$ with the property that $v\left(\chi^{\lambda}\right)=\langle\lambda, w\rangle$ for every $\lambda$ in $N$.

(3) For every $f \in I$, the polynomial $\operatorname{in}_{w} f$ is not a monomial.

(4) The ideal $\operatorname{in}_{w} I$ does not contain any monomial.

(5) The scheme $\operatorname{in}_{w} C$ is nonempty. 
Note that in conditions (3) and (4), zero is not considered a monomial.

Proof. The equivalence of (1), (3) and (4) is Theorem 2.1 of [Speyer and Sturmfels 2004]. The proof there that (4) implies (1) is flawed; see [Draisma 2008; Payne 2009b] for corrections and improvements. The equivalence of (4) and (5) is simply the Nullstellensatz - since monomials are units in $\kappa[M]$, the ideal in $_{w} I$ contains a monomial if and only if it is all of $\kappa[M]$. The equivalence of (1) and (2) is Theorem 2.2.5 of [Einsiedler et al. 2006].

Define the subset of $\mathbb{Q} \otimes N$ where any of the equivalent conditions above holds to be Trop $C$. Note that condition (3) of the above proposition clearly singles out a closed set. If $\mathbb{K}$ is a power series field and $C$ has constant coefficients, then we can define Trop $C \subset \mathbb{R}^{n}$ using the definition of $\operatorname{in}_{w} f$ which is defined for $f \in \kappa[M]$. There is little risk of confusion in defining Trop $C$ both for $C \subset \mathbb{T}(\mathbb{K}, N)$ and $C \subset \mathbb{T}(\kappa, N)$. The precise relation is as follows: Let $\mathbb{K}^{\prime}$ be any power series field, with associated notation $\left(\mathbb{K}^{\prime}, v^{\prime}, \mathcal{O}^{\prime}, M^{\prime}, \kappa^{\prime}\right)$ such that $\kappa^{\prime}=\kappa$, and let $C$ be a closed subscheme of $\mathbb{T}(\kappa, N)$. Then $\operatorname{Trop} C=\operatorname{Trop}\left(C \times_{\operatorname{Spec} \kappa^{\prime}} \operatorname{Spec} \mathbb{K}^{\prime}\right)$. The next proposition summarizes basic results on the structure of Trop $C$.

Proposition 2.3. Let $C \subset \mathbb{T}(\mathbb{K}, N)$. Then Trop $C$ can be given the structure of a polyhedral complex (with finitely many faces). Furthermore, we may do this in such $a$ way that, for $\sigma$ any face of this polyhedral complex and $w$ and $w^{\prime}$ two points in the relative interior of $\sigma$, we have $\operatorname{in}_{w} C=\operatorname{in}_{w^{\prime}} C$. If $C$ is $d$-dimensional then Trop $C$ has dimension $d$. If $C$ is pure of dimension $d$ then so is Trop $C$. If $C$ is connected then Trop $C$ is connected. If $C$ is connected in codimension one and $\mathbb{K}$ has characteristic zero then Trop $C$ is connected in codimension one.

If $\mathbb{K}$ is a power series field and $C$ has constant coefficients, then Trop $C$ can be given the structure of a polyhedral fan.

Proof. The existence of the polyhedral structure is proved by Bieri and Groves [1984] using description (2) of Trop $C$. The claim about initial ideals is proved by Sturmfels [2002] in a slightly more specialized context in the course of proving his Theorem 9.6. The dimensionality claim is also proven in [Bieri and Groves 1984] and is proven by a different method (under more restrictive hypotheses than we adopt here) in [Sturmfels 2002]. Sturmfels proves the pureness claim as well. The connectivity result is proven in [Einsiedler et al. 2006]. The connectivity in codimension one is proven in [Bogart et al. 2007]. That proof is given in a somewhat more restrictive setting than we have adopted here, but there is no difficulty in extending the arguments.

We will call a polyhedral subdivision of Trop $C$ a good subdivision if, whenever $w$ and $w^{\prime}$ are two points in the relative interior of the same face $\sigma$, we have $\operatorname{in}_{w} C=\operatorname{in}_{w^{\prime}} C$. So one of the parts of the above proposition is that Trop $C$ always has a good subdivision. 
Let $C$ be a closed subscheme of $\mathbb{T}(\mathbb{K}, N)$, and suppose that we have equipped Trop $C$ with a good subdivision, for which $\sigma$ is a $k$-dimensional face. Let $w$ lie in the relative interior of $\sigma$. Let $H(\sigma) \subset \mathbb{R} \otimes N$ be the vector space spanned by $w_{1}-w_{2}$, for $w_{1}, w_{2} \in \sigma$, and let $\exp (H(\sigma))$ be the corresponding subtorus of $\mathbb{T}(\kappa, N)$.

Proposition 2.4. With the above notation, $\operatorname{in}_{w}(C)$ is invariant for the action of $\exp (H(\sigma))$.

Proof. Let $v \in H(\sigma) \cap N$ and let $\epsilon$ be a small rational number. Then, from Lemma 2.1, $\operatorname{in}_{w+\epsilon v} C=\operatorname{in}_{v} \operatorname{in}_{w} C$ and, by the definition of a good subdivision and $H(\sigma)$, we have $\operatorname{in}_{w+\epsilon v} C=\operatorname{in}_{w} C$. So $\operatorname{in}_{v} \operatorname{in}_{w} C=\operatorname{in}_{w} C$. The left-hand side is invariant for the one-dimensional subgroup $\exp (\mathbb{R} v)$, so we have shown that $\operatorname{in}_{w} C$ is invariant under $\exp (\mathbb{R} v)$ for any $v \in H(\sigma) \cap N$.

In particular, in the above setting, suppose that $C$ is pure of dimension $d$ and $k=d$. Then $\operatorname{in}_{w} C / \exp (H(\sigma))$ is zero-dimensional. We define $\mu(w)=m(\sigma)$ to be the length of this scheme over $\kappa$.

\section{Statement of results}

We now have enough tropical background to state our main results. We need one combinatorial definition:

Let $\Gamma$ be a finite graph. We write $\partial \Gamma$ for the set of degree-one vertices of $\Gamma$. Let $\iota$ be a continuous map $\Gamma \backslash \partial \Gamma \rightarrow \mathbb{Q} \otimes N$ such that an edge $e$ of $\Gamma$ is taken to

(1) either a finite line segment or a point if neither endpoint of $e$ is in $\partial \Gamma$,

(2) an unbounded ray if one endpoint of $e$ is in $\partial \Gamma$, and

(3) a line if both ends of $e$ are in $\partial \Gamma$.

We consider such pairs $(\iota, \Gamma)$ up to reparameterization of the edges of $\Gamma$. By our conventions on rationality of polyhedral complexes, $\iota(e)$ has slope in $N$ for every edge $e$ of $\Gamma$. If $v$ is a vertex of $\Gamma$, and $e$ is an edge of $\Gamma$ with an endpoint at $v$, then we write $\rho_{v}(e)$ for the minimal lattice vector parallel to $\iota(e)$ which points in the direction away from $\iota(v)$. (If $v$ is in $\partial \Gamma$, so that $\iota(e)$ is an unbounded ray or line, then $\iota(v)$ should be thought of as "at infinity", so $\rho_{v}(e)$ is negative the direction in which $\iota(e)$ goes to infinity.) If $e$ is mapped to a point, define $\rho_{v}(e)$ to be 0 . Suppose that $m$ is a function assigning a positive integer to each edge of $\Gamma$. We say that $(\iota, \Gamma, m)$ is a zero-tension curve if, for every vertex $v$ in $\Gamma \backslash \partial \Gamma$, we have $\sum_{e \ni v} m(e) \rho_{v}(e)=0$. We introduce the notation $\sigma_{v}(e)$ for $m(e) \rho_{v}(e)$. If $(\iota, \Gamma, m)$ is any zero-tension curve, we place a metric ${ }^{4}$ on $\Gamma \backslash \partial \Gamma$. For any finite edge $e$, set

\footnotetext{
${ }^{4}$ This might be only a pseudometric; if $\iota$ collapses an edge of $\Gamma$, then we have $d(x, y)=0$ for some $x$ and $y$ which are not equal. This will not be a difficulty.
} 
the length of $e$ to be $\ell$, where the endpoints of $\iota(e)$ differ by $\ell \rho_{v}(e)$. If one or both of the endpoints of $e$ are in $\partial \Gamma$, then we define $e$ to have infinite length.

We define a partial zero-tension curve to be a triple $(\iota, \Gamma, m)$ which obeys the above conditions except that if one endpoint of an edge $e$ is in $\partial \Gamma$, we permit that edge to be taken to a finite line segment rather than a ray. Partial zero-tension curves are analogous to analytic maps from a Riemann surface with holes of positive area; zero-tension curves, which will be our main concern, are analogous to algebraic maps from punctured Riemann surfaces.

We define the genus of a zero-tension curve to be the first Betti number of $\Gamma$. We define the degree of a zero-tension curve as follows: Let $D \subset N$ be the (finite) set of values assumed by $-\rho_{v}(e)$ as $v$ ranges through $\partial \Gamma$. For each $\lambda \in D$, let $m_{\lambda}=\sum m(e)$, where the sum is over $e$ with an endpoint $v$ in $\partial \Gamma$ and $-\rho_{v}(e)=\lambda$. Then the degree of $(\iota, \Gamma, m)$ is the set $\left\{m_{\lambda} \cdot \lambda: \lambda \in D\right\}$. We now state that, given a curve $C$, the polyhedral complex Trop $C$ reflects the degree and genus of the curve.

Theorem 3.1. Let $C$ be a connected (punctured) curve of genus $g$ over $\mathbb{K}$ equipped with a map $\phi: C \rightarrow \mathbb{T}(\mathbb{K}, N)$. Let $\delta \subset N$ be the degree of $(C, \phi)$. Then there is a connected zero-tension curve $(\iota, \Gamma, m)$ of degree $\delta$ and genus at most $g$ with $\iota(\Gamma)=$ Trop $\phi(C)$. Moreover, choose a good subdivision of $\operatorname{Trop} \phi(C)$ and subdivide $\Gamma$ by the preimage of this subdivision. Then the weights $\mu$ on Trop $C$ and $m$ on $\Gamma$ are related by $\mu(w)=\sum_{\iota(e) \ni w} m(w)$.

Extend $C$ to a flat family over Spec 0 whose fiber over Spec $\kappa$ consists of smooth reduced curves glued along nodes. ${ }^{5}$ If any of the components of the $\kappa$-fiber are not rational, then we can take the genus of $\Gamma$ to be strictly less than $g$.

Theorem 3.1 is implicit in the work of many authors, beginning with Grigory Mikhalkin. A complete proof is given in [Nishinou and Siebert 2006]; we explain how to find this theorem in that work: Proposition 6.3 of [Nishinou and Siebert 2006] states that, given $C$ and $\phi$, there is degeneration of $\mathbb{T}(\mathbb{K}, N)$ (over Spec $\boldsymbol{O}$ ) to a union of toric varieties and a degeneration of $C$ (over Spec $\mathcal{O}$ ) to a nodal curve so that $\phi$ extends on the $\operatorname{Spec} \kappa$ fiber to a torically transverse stable map. Write $C_{0}$ for the nodal curve and $\phi_{0}$ for the map from $C_{0}$. In the course of proving Theorem 8.3, Nishinou and Siebert verify that $\left(C_{0}, \phi_{0}\right)$ is an object they call a pre-log curve. In Construction 4.4, they explain how to build a zero-tension curve $(\iota, \Gamma, m)$ from a pre-log curve. The components of $C_{0}$ are organized in equivalence classes, where members of the same class are called "indistinguishable". The union of the components in each equivalence class is connected. The vertices of $\Gamma$ correspond to these classes of $C_{0}$, and the edges of $\Gamma$ to nodes of $C_{0}$ connecting components in different classes. Since $C_{0}$ is a stable degeneration of a genus- $g$

\footnotetext{
${ }^{5}$ For example, extend $C$ to a family of stable curves.
} 
curve, the graph $\Gamma$ may have first Betti number at most $g$. This establishes the first paragraph of the theorem.

For the second paragraph, let $C_{0}^{\prime}$ be the $\kappa$-fiber of the family described in the second paragraph of the theorem. Let $Y$ be the unique stable limit of $C /$ Spec $\mathbb{K}$. Then $Y$ is obtained from $C_{0}^{\prime}$ by collapsing some curve of genus zero. So if $C_{0}^{\prime}$ has any nonrational components, so does $Y$. But $Y$ is also obtained from $C_{0}$ by collapsing some genus-zero components, so if $C_{0}^{\prime}$ has nonrational components then so does $C_{0}$, and thus $\Gamma$ must have first Betti number strictly less than $g$.

We now state the main results of the paper.

Theorem 3.2. Let $(\iota, \Gamma, m)$ be a zero-tension curve of genus zero and degree $\delta$. Then there is a (punctured) genus-zero curve $C$ over $\mathbb{K}$ and a map $\phi: C \rightarrow \mathbb{T}(\mathbb{K}, N)$ such that $(C, \phi)$ has degree $\delta$ and $\iota(\Gamma)=\operatorname{Trop} \phi(C)$. The edge weighting $m$ is related to the multiplicities $\mu$ as described in Theorem 3.1.

Theorem 3.2 was previously proven, by methods of log geometry, in [Nishinou and Siebert 2006].

Theorem 3.3. Let $(\iota, \Gamma, m)$ be a zero-tension curve of genus one and degree $\delta$. Let $e_{1}, \ldots, e_{r}$ be the edges of the unique circuit of $\Gamma$. Assume that the slopes of $\iota\left(e_{1}\right), \ldots, \iota\left(e_{r}\right)$ span $\mathbb{Q} \otimes N$. Then there is a (punctured) genus-one curve $C$ over $\mathbb{K}$ and a map $\phi: C \rightarrow \mathbb{T}(\mathbb{K}, N)$ such that $(C, \phi)$ has degree $\delta$ and $\iota(\Gamma)=\operatorname{Trop} \phi(C)$. The edge weighting $m$ is related to the multiplicities $\mu$ as described in Theorem 3.1.

Mikhalkin [2006, Theorem 1] states without proof a result which includes both Theorems 3.2 and 3.3.

The importance of the criterion that the slopes of the edges $\iota\left(e_{1}\right), \ldots, \iota\left(e_{r}\right)$ span $\mathbb{Q} \otimes N$ was first pointed out by Mikhalkin. Following Mikhalkin, we say that $(\iota, \Gamma, m)$ is ordinary when this condition holds, and superabundant when it does not. ${ }^{6}$ When dealing with superabundant curves, we need to impose a further criterion, which is original to this paper. Let $(\iota, \Gamma, m)$ be a zero-tension curve of genus one and degree $\delta$. Let $e_{1}, \ldots, u_{r}$ be the edges of the unique circuit of $\Gamma$. Let $H$ be an affine hyperplane in $\mathbb{Q} \otimes N$ containing all of the line segments $\iota\left(e_{i}\right)$, but not containing the entire curve $\iota(\Gamma)$. Let $\Delta$ be the connected component of $\Gamma \cap \iota^{-1}(H)$ which contains the circuit of $\Gamma$ and let $x_{1}, \ldots, x_{s}$ be the vertices of $\Delta$ which are also in the (topological) closure of $\Gamma \backslash \Delta$; we call these the boundary vertices of $\Delta$. Let $d_{1}, \ldots, d_{s}$ be the distances from $x_{1}, \ldots, x_{s}$ to the nearest point on the circuit of $\Gamma$. Then we say that $(\iota, \Gamma, m)$ is well spaced with respect to $H$ if the minimum of the numbers $\left(d_{1}, \ldots, d_{s}\right)$ occurs more than once. We say that

\footnotetext{
${ }^{6}$ The motivation for this terminology is that one can define a space of deformations of $(\iota, \Gamma, m)$. A deformation consists of changing the lengths of the edges of $\Gamma$, while keeping the slopes of the images of those edges constant. One can show that ordinary curves have deformation spaces of the "expected dimension", while the deformation space of superabundant curves is larger than expected.
} 
$(\iota, \Gamma, m)$ is well spaced if it is well spaced with respect to every $H$ containing the circuit of $\Gamma$.

Theorem 3.4. Assume that $\kappa$ has characteristic zero. Let $(\iota, \Gamma, m)$ be a zero-tension curve of genus one and degree $\delta$ and assume that $(\iota, \Gamma, m)$ is well spaced. Then there is a (punctured) genus-one curve $C$ over $\mathbb{K}$ and a map $\phi: C \rightarrow \mathbb{T}(\mathbb{K}, N)$ such that $\iota(\Gamma)=\operatorname{Trop} C$.

There is a partial converse to this theorem; see Proposition 9.2.

It is possible to prove enumerative versions of all of these results, where we count curves $(C, \phi)$ with $\operatorname{Trop} \phi(C)=\iota(\Gamma)$ that meet subvarieties of $\mathbb{T}(\mathbb{K}, N)$. We do not do so here. Partially, this is because it would add greatly to the length of the exposition. A more important reason is that, as described in the introduction, we have no results regarding the lifting of zero-tension curves of genus zero to actual curves of genus one and therefore we do not know how to productively apply such enumerative results.

In a sequel to this paper I will establish analogous results for curves of genus greater than one.

\section{The Bruhat-Tits tree}

We will spend the rest of this paper proving Theorems 3.2, 3.3 and 3.4. One of our main technical tools is the Bruhat-Tits tree. A good reference for our discussion is Chapter 2 of [Morgan and Shalen 1984]. A more sophisticated approach here would be to replace the Bruhat-Tits tree by the analytification of $\mathbb{P}^{1}(\mathbb{K})$ in the sense of Berkovich. Recently, several authors have begun fully using the Berkovich technology for tropical purposes; the interested reader should begin with [Payne 2009a; Baker et al. 2012]. In this paper, we will restrict ourselves to the more concrete Bruhat-Tits tree.

We denote by BT( $(\mathbb{K})$ the set of $\mathcal{O}$-submodules of $\mathbb{K}^{2}$ which are isomorphic to $\mathbb{O}^{2}$, modulo $\mathbb{K}^{*}$-scaling. We write $\bar{M}$ for the equivalence class of a module $M$. We equip BT $(\mathbb{K})$ with the metric where $d\left(\bar{M}_{1}, \bar{M}_{2}\right)$ is the minimum of the set of $\epsilon$ such that there exists an $\alpha$ with $M_{1} \supseteq t^{\alpha} M_{2} \supseteq t^{\alpha+\epsilon} M_{1}$; this minimum exists and is independent of the choice of representatives $M_{1}$ and $M_{2}$. Clearly, $d\left(\bar{M}_{1}, \bar{M}_{2}\right)$ is always in $\mathbb{Q}$. The metric space BT( $\mathbb{K})$ is called the Bruhat-Tits tree of $\mathbb{K}$.

If we made the analogous construction working over $\boldsymbol{K}$, we could equip BT with the structure of the vertices of a tree so that distance was the graph-theoretic distance. Instead, BT $(\mathbb{K})$ is what is called a $\mathbb{Q}$-tree (see [Morgan and Shalen 1984]). We remind our reader of the convention that all metric trees have edges whose lengths are in $\mathbb{Q}$, and the points of such a tree are the points whose distances from the vertices are rational. Being a $\mathbb{Q}$-tree is a more general concept than this; the following proposition lists the "tree-like" properties of $\mathbb{Q}$. 
Proposition 4.1. If $\bar{M}_{1}$ and $\bar{M}_{2} \in \mathrm{BT}(\mathbb{K})$ with $d\left(\bar{M}_{1}, \bar{M}_{2}\right)=d$, then there is a unique distance-preserving map $\phi:[0, d] \cap \mathbb{Q} \rightarrow \mathrm{BT}(\mathbb{K})$ with $\phi(0)=\bar{M}_{1}$ and $\phi(d)=\bar{M}_{2}$. Explicitly, if $t^{\alpha} M_{1} \supseteq M_{2} \supseteq t^{\alpha+d} M_{1}$, then $\phi(e)=\overline{t^{\alpha+e} M_{1}+M_{2}}$. We will call the image of $\phi$ the path from $\bar{M}_{1}$ to $\bar{M}_{2}$ and denote it by $\left[\bar{M}_{1}, \bar{M}_{2}\right]$. If $\bar{M}_{1}, \ldots, \overline{M_{n}} \subset \mathrm{BT}(\mathbb{K})$, then $\bigcup_{i \neq j}\left[\overline{M_{i}}, \overline{M_{j}}\right]$ is a metric tree.

Suppose now that $\left(x_{1}: y_{1}\right)$ and $\left(x_{2}: y_{2}\right)$ are distinct members of $\mathbb{P}^{1}(\mathbb{K})$. Then we can similarly define a map $\phi: \mathbb{Q} \rightarrow \mathrm{BT}(\mathbb{K})$ by $\phi(e)=\overline{\mathbb{O}\left(x_{1}, y_{1}\right)+t^{e} \mathbb{O}\left(x_{2}, y_{2}\right)}$. We will call the image of this $\phi$ the path from $\left(x_{1}: y_{1}\right)$ to $\left(x_{2}: y_{2}\right)$ and denote it by $\left[\left(x_{1}: y_{1}\right),\left(x_{2}: y_{2}\right)\right]$. (The specific map $\phi: \mathbb{Q} \rightarrow \mathrm{BT}(\mathbb{K})$ depends on the choice of representatives $\left(x_{1}, y_{1}\right)$ and $\left(x_{2}, y_{2}\right)$, but the image $\phi(\mathbb{Q})$ does not.) Similarly, if $\bar{M} \in \mathrm{BT}(\mathbb{K})$ and $(x: y) \in \mathbb{P}^{1}(\mathbb{K})$, we can define a semi-infinite path from $(x: y)$ to $\bar{M}$ denoted by $[(x: y), \bar{M}]$. We introduce the notation $\overline{\mathrm{BT}}(\mathbb{K})$ for $\mathrm{BT}(\mathbb{K}) \cup \mathbb{P}^{1}(\mathbb{K})$.

If $Z$ is a subset of $\overline{\mathrm{BT}}(\mathbb{K})$, we denote by $[Z]$ the subspace $\bigcup_{z, z^{\prime} \in Z}\left[z, z^{\prime}\right]$ of $\overline{\mathrm{BT}}(\mathbb{K})$. For simplicity, assume that $|Z| \geq 3$. If $Z$ is finite, then $[Z]$ is a metric tree with a semi-infinite ray for each member of $Z \cap \mathbb{P}^{1}(\mathbb{K})$. We will say that this ray has its end at the corresponding member of $Z \cap \mathbb{P}^{1}(\mathbb{K})$. We will abbreviate $\left[\left\{z_{1}, \ldots, z_{n}\right\}\right]$ as $\left[z_{1}, \ldots, z_{n}\right]$.

The case where $Z$ is a four-element subset of $\mathbb{P}^{1}(\mathbb{K})$ is of particular importance for us. Let $\{w, x, y, z\} \subset \mathbb{P}^{1}(\mathbb{K})=\mathbb{K} \cup\{\infty\}$. We define the cross ratio $c(w, x: y, z)$ by

$$
c(w, x: y, z)=\frac{(w-y)(x-z)}{(w-z)(x-y)} .
$$

Note that $c(w, x: y, z)=c(x, w: z, y)=c(y, z: w, x)=c(z, y: x, w)$ and $c(w, x: y, z)=c(w, x: z, y)^{-1}$.

Proposition 4.2. The metric space $[w, x, y, z]$ is a metric tree with 4 semi-infinite rays and either 1 or 2 internal vertices.

If $[w, x, y, z]$ has 2 internal vertices, let $d$ be the length of the internal edge and suppose that the rays ending at $w$ and $x$ lie on one side of that edge and the rays through $y$ and $z$ on the other. Then

$$
\begin{aligned}
& v(c(w, x: y, z))=0, \\
& v(c(w, y: x, z))=-v(c(w, y: z, x))=d, \\
& v(c(w, z: x, y))=-v(c(w, z: y, x))=d .
\end{aligned}
$$

The first statement can be strengthened to say that $v(c(w, x: y, z)-1)=d$. (The valuations of all other permutations of $\{w, x, y, z\}$ can be deduced from these by the symmetries of the cross ratio.)

If $[\{w, x, y, z\}]$ has only 1 internal vertex, then

$$
v(c(w, x: y, z)-1)=v(c(w, x: y, z))=0,
$$


and the same holds for all permutations of $\{w, x, y, z\}$.

This proposition can be remembered as saying " $v(c(w, x: y, z))$ is the signed length of $[w, x] \cap[y, z]$ ", where the sign tells us whether the two paths run in the same direction or the opposite direction along their intersection.

Proof. The group $\mathrm{GL}_{2}(\mathbb{K})$ acts on $\overline{\mathrm{BT}}(\mathbb{K})$ through the action on $\mathbb{K}^{2}$. It is well known that $c$ is $\mathrm{GL}_{2}(\mathbb{K})$ invariant, and the definitions of $[x, y]$ and the metric on $\mathrm{BT}(\mathbb{K})$ are clearly $\mathrm{GL}_{2}(\mathbb{K})$ equivariant. So the whole theorem is invariant under $\mathrm{GL}_{2}(\mathbb{K})$ and we may use this action to take $w, x$ and $y$ to 0,1 and $\infty$.

Our hypothesis in the second paragraph of the proposition is that $[0,1, \infty, z]$ is a tree with 0 and 1 on one side of a finite edge of length $d$ and $z$ and $\infty$ on the other. It is easy to check that this is equivalent to requiring that $v(z)=-d<0$. Then

$$
c(0,1: \infty, z)=1-\frac{1}{z},
$$

which does indeed have valuation 0 , and $c(0,1: \infty, z)-1=-1 / z$, which does indeed have valuation $d$. Similarly, $c(0, \infty: 1, z)=1 / z$, which has valuation $d$, and $c(0, z: 1, \infty)=1 /(1-z)$, which has valuation $d$.

In the third paragraph of the proposition, the assumption that the tree has no finite edge implies that $v(z)=v(z-1)=0$. The argument then continues as before.

\section{Lemmas on zero-tension curves}

We pause to prove two combinatorial lemmas about zero-tension curves.

Lemma 5.1. Let $(\iota, \Gamma, m)$ be a connected partial ${ }^{7}$ zero-tension curve in $\mathbb{Q}^{n}$. Suppose that $\iota(\Gamma)$ is not contained in any hyperplane. Then the set of vectors $\sigma_{v}(e)$, where $v$ runs over $\partial \Gamma$, spans $\mathbb{Q}^{n}$.

Proof. Suppose, for the sake of contradiction, that there is some nonzero $\lambda \in \mathbb{Q}^{n}$ with $\left\langle\lambda, \sigma_{v}(e)\right\rangle=0$ for every degree-one vertex $v$ of $\Gamma$. Let $h(u)$ be the function $\langle\lambda, \iota(u)\rangle$ on $\Gamma \backslash \partial \Gamma$. As $h$ is constant on every ray of $\Gamma$ ending at a degree-one vertex, and in particular on all of the unbounded rays of $\Gamma$, the function $h$ is bounded on $\Gamma$. Let $U$ be the (nonempty) subset of $\Gamma$ on which $h$ achieves its maximum. Clearly, $U$ is closed.

We now show that $U$ is also open. Clearly, if $U$ contains a point $p$ in the interior of an edge of $\Gamma$, then it contains that entire edge and, in particular, $U$ contains an open neighborhood of $p$. So we just need to show that, if $u$ is a vertex of $\Gamma$ contained in $U$, then $U$ contains an open neighborhood of $u$. If $u$ is a degree-one vertex of $\Gamma$, then $h$ is constant in a neighborhood of $u$ by our hypothesis. If $u$ is not a degree-one vertex, then by the zero-tension condition, we have $\sum_{e \ni u}\left\langle\lambda, \sigma_{u}(e)\right\rangle=0$.

\footnotetext{
${ }^{7}$ Recall that the adjective partial means we permit edges which end at a vertex of $\partial \Gamma$ to be taken to a finite line segment rather than to an infinite ray.
} 
Since we have assumed that $h$ is maximized at $u$, we have $\left\langle\lambda, \sigma_{u}(e)\right\rangle \leq 0$ for every edge $e$ containing $u$ and we conclude that $h$ is constant in a neighborhood of $u$, as desired.

So $U$ is open, closed and nonempty. As $\Gamma$ is connected, $U=\Gamma$ and we have that $h$ is constant on $\Gamma$. This contradicts our assumption that $\iota(\Gamma)$ is not contained in any hyperplane.

Morally, this proof is an instance of the "tropical maximum modulus principle". The linear functional defining $H$ is a harmonic function on $\Gamma$ in the sense of [Baker and Norine 2007; Gathmann and Kerber 2008] and other papers, and we are showing that if it is bounded, then it is constant.

One difficulty with Theorem 3.1 is that it states that a zero-tension curve with $\iota(\Gamma)=$ Trop $C$ exists, but it doesn't help us choose from among several possible candidates for $(\iota, \Gamma, m)$. We now introduce a concept that will let us guarantee that essentially only one such $(\iota, \Gamma, m)$ exists. We define $(\phi, C)$ to be trivalent if, for some (equivalently any) good subdivision of Trop $\phi(C)$, we have $m_{e}=1$ for every edge $e$ and the degree of $v$ is at most 3 for every vertex $v$.

Lemma 5.2. Let $(\phi, C)$ be a trivalent curve and $(\iota, \Gamma, m)$ a zero-tension curve with $\iota(\Gamma)=\operatorname{Trop} \phi(C)$, such that if $e$ is any edge of (a good subdivision of ) Trop $\phi(C)$, then we have $m_{e}=\sum_{\iota(f)=e} m_{f}$. (The sum is over edges $f$ of $\Gamma$ mapping to e.)

Than there is a subgraph $\Gamma^{\prime}$ of $\Gamma$ which maps isomorphically onto Trop $\phi(C)$. Specifically, we take $\Gamma^{\prime}$ to be the union of all edges of $\Gamma$ which are not contracted to points under $\iota$. In particular, if ı contracts no edge, then $\Gamma \cong$ Trop $C$.

Proof. First, we note that if $e$ is an edge of Trop $\phi(C)$, then each point in the interior of $e$ can have only one preimage in $\Gamma$, by the equation $1=m_{e}=\sum_{\iota(f)=e} m_{f}$. Next, let $x$ be a vertex of Trop $\phi(C)$ with edges $e_{1}, e_{2}$ and possibly $e_{3}$ coming out of $x$. Let $y \in \Gamma^{\prime}$ be a preimage vertex of $x$. Then there must be edges leaving $y$ which map down to each of the $e_{i}$, as otherwise the zero-tension condition would be violated. (If all of the edges leaving $y$ map down to a point, then $y$ is not in $\Gamma^{\prime}$.) Then there can be no other vertex $z$ of $\Gamma^{\prime}$ which maps to $x$, as there are no edges of Trop $\phi(C)$ left for the edges coming from $z$ to map to.

So every vertex of Trop $\phi(C)$ and the interior of every edge of Trop $\phi(C)$ has only one preimage in $\Gamma^{\prime}$; that is, the restriction of $\iota$ to $\Gamma^{\prime}$ is bijective onto its image. Moreover, $\Gamma^{\prime}$ is closed in $\Gamma$, so the map $\Gamma^{\prime} \rightarrow$ Trop $\phi(C)$, like the map $\Gamma \rightarrow \operatorname{Trop} \phi(C)$, is a closed map. But we know $\Gamma^{\prime} \rightarrow \operatorname{Trop} \phi(C)$ is bijective, so this is also an open map. A continuous open bijective map is a homeomorphism.

\section{Tropical curves of genus zero}

The aim of this section is to prove Theorem 3.2. This result appears in [Nishinou and Siebert 2006] and will also appear in a future publication of Mikhalkin. Our 
method of proof is not only more explicitly constructive than these, but will also preview many of the methods which we will use to deal with higher-genus curves. Let $(\iota, \Gamma, m)$ be a zero-tension curve with $\Gamma$ a tree. From now until the end of the paper, fix an identification of $N$ with $\mathbb{Z}^{n}$.

Proposition 6.1. Let $T$ be a metric tree with finitely many vertices. Then there is a subset $Z$ of $\overline{\mathrm{BT}}(\mathbb{K})$ such that $[Z]$ is isometric to $T$. If every leaf of $T$ is at the end of an unbounded edge, then we can take $Z \subset \mathbb{P}^{1}(\mathbb{K})$.

Proof. Our proof is by induction on the number of finite-length edges of $T$. If $T$ has $l \geq 3$ leaves and no finite edges, then $T$ is isometric to $\left[z_{1}, \ldots, z_{l}\right]$ for $\left\{z_{1}, \ldots, z_{l}\right\}$ any $l$ elements of $\mathbb{K}^{*}$ with valuation 0 and distinct images in $\kappa^{*}$. If $T$ is an unbounded edge which is infinite in both directions then $T \cong[0, \infty]$; if $T$ is an unbounded ray which is infinite in one direction then $T=\left[0^{2}, \infty\right]$; if $T$ is a point then $T \cong\left[\mathrm{O}^{2}\right]$. These are all of the cases with no finite edges, so our base case is complete.

Now let $e$ be a finite edge of $T$ of length $d$ joining vertices $v_{1}$ and $v_{2}$. Remove $e$ from $T$, separating $T$ into two trees $T_{1}$ and $T_{2}$. Define trees $T_{s}^{\prime}$, where $s=1,2$, by adding an unbounded edge to $T_{s}$ at $v_{s}$. By induction, we can find subsets $Z_{1}$ and $Z_{2} \subset \overline{\mathrm{BT}}(\mathbb{K})$ with $\left[Z_{s}\right]$ isometric to $T_{s}^{\prime}$. Let $z_{s} \in Z_{s}$ be the element of $Z_{s}$ at the end of the new ray added to $T_{s}$.

Without loss of generality, we may assume that $z_{1}=0$ and $z_{2}=\infty$. Furthermore, we may translate $T_{1}^{\prime}$, preserving 0 , so that $v_{1}$ lies on $[0, \infty]$, and similarly, we may translate $T_{2}^{\prime}$, preserving $\infty$, so that $v_{2}$ lies on $[0, \infty]$. By multiplying $Z_{1}$ and $Z_{2}$ by elements of $\mathbb{K}^{*}$, we may assume that these points lie distance $d$ apart with $v_{2}$ closer to $z_{1}$ than $v_{1}$ is. Then $T$ is isometric to $\left[\left(Z_{1} \backslash\left\{z_{1}\right\}\right) \cup\left(Z_{2} \backslash\left\{z_{2}\right\}\right)\right]$.

If all of the leaves of $T$ were at the end of unbounded edges, then this would also be true of $T_{1}^{\prime}$ and $T_{2}^{\prime}$, and tracing through the proof, we see that $Z \subset \mathbb{P}^{1}(\mathbb{K})$.

Recall that we have been given a zero-tension curve $(\iota, \Gamma, m)$ of genus zero and we want to construct an actual genus-zero curve with $\iota(\Gamma)$ as its tropicalization. Let $Z \subset \mathbb{P}^{1}(\mathbb{K})$ be such that $[Z]$ is isometric to $\Gamma$. We define multisets $Z_{1}^{+}, \ldots, Z_{n}^{+}, Z_{1}^{-}, \ldots, Z_{n}^{-}$as follows: All of the elements of $Z_{i}^{ \pm}$lie in $Z$. Let $z \in Z$ correspond to the end of an infinite ray $e$ of $\Gamma$. Suppose that $\sigma_{z}(e)=\left(s_{1}, \ldots, s_{n}\right)$. Then $z \in Z_{i}^{ \pm}$if and only if $\pm s_{i}<0$. In this case, the number of times that $z$ occurs in $Z_{i}^{ \pm}$is $\left|s_{i}\right|$. Let $\phi_{i}$ be a rational function on $\mathbb{P}^{1}$ with zeroes at the points of $Z_{i}^{+}$and poles at the points of $Z_{i}^{-}$. (If $\infty$ is not in $Z_{i}^{+}$or $Z_{i}^{-}$, we may take $\phi_{i}(u)=\prod_{z \in Z_{i}^{+}}(u-z) / \prod_{z \in Z_{i}^{-}}(u-z)$.) Define a rational map $\phi: \mathbb{P}^{1}(\mathbb{K}) \rightarrow \mathbb{K}^{n}$ by the formula $\phi(u)=\left(\phi_{1}(u), \ldots, \phi_{n}(u)\right)$. Here $u$ is a coordinate on $\mathbb{P}^{1}(\mathbb{K})$, thought of as $\mathbb{K} \cup\{\infty\}$.

The following theorem states that $C$ and $\phi$ satisfy the conditions on the curve and the map in Theorem 3.2; the conclusion of Theorem 3.2 follows. 
Theorem 6.2. The curve $\phi\left(\mathbb{P}^{1}(\mathbb{K})\right)$ is a genus-zero curve of degree the degree of $(\iota, \Gamma, m)$. Trop $\phi\left(\mathbb{P}^{1}(\mathbb{K})\right)$ is a translation of $\iota(\Gamma)$. Thus, by rescaling the $\phi_{i}(u)$ by elements of $\mathbb{K}^{*}$, we can arrange that $\operatorname{Trop} \phi\left(\mathbb{P}^{1}(\mathbb{K})\right)$ is $\iota(\Gamma)$.

From now until the end of the proof, we identify $[Z]$ with $\Gamma$ so that we can write $\iota:[Z] \rightarrow \mathbb{Q}^{n}$.

Proof. The curve $\phi\left(\mathbb{P}^{1}(\mathbb{K})\right)$ clearly has genus zero. It has the same degree as $(\iota, \Gamma, m)$ because, by Proposition 1.2 , the degree can be computed simply by looking at the orders of vanishing of the coordinate functions on $\left(\mathbb{K}^{*}\right)^{n}$ at the points of $\mathbb{P}^{1}$ where $\phi$ is not defined. We built $\phi$ to have exactly the required zeroes and poles. We now move to the interesting point, the claim that $\operatorname{Trop} \phi\left(\mathbb{P}^{1}(\mathbb{K})\right)$ is a translation of $\iota(\Gamma)$.

Let $u \in \mathbb{P}^{1}(\mathbb{K}) \backslash Z$. Then $[Z]$ is a tree and $[Z \cup\{u\}]$ is a tree with one additional end. Let $b(u)$ be the point of $[Z]$ at which that end is attached. (In Figure 1, [Z] is shown in solid lines, the points of $Z$ are represented by $z$ 's, the path from $u$ to $b(u)$ is dashed, and $b(u)$ is the solid dot.) We claim that, up to a translation, $v(\phi(u))$ is $\iota(b(u))$. In other words, if $u_{1}$ and $u_{2}$ are distinct members of $u \in \mathbb{P}^{1}(\mathbb{K}) \backslash Z$, we must show that for each $i$ between 1 and $n$ we have

$$
v\left(\phi_{i}\left(u_{1}\right)\right)-v\left(\phi_{i}\left(u_{2}\right)\right)=\iota\left(b\left(u_{1}\right)\right)_{i}-\iota\left(b\left(u_{2}\right)\right)_{i} .
$$

It is enough to show this in the case where $b\left(u_{1}\right)$ and $b\left(u_{2}\right)$ lie in the same edge $e$ of $[Z]$. Let $\sigma_{u_{2}}(e)=\left(s_{1}, \ldots, s_{n}\right)$. We will fix one coordinate $i$ to pay attention to, so $i$ will not appear in our notation. Let $Z_{i}^{+}=\left\{z_{1}^{+}, \ldots, z_{r}^{+}\right\}$and $Z_{i}^{-}=\left\{z_{1}^{-}, \ldots, z_{r}^{-}\right\}$. We may find constants $1 \leq s^{+}, s^{-} \leq n$ and order the $z_{j}^{ \pm}$such that $z_{j}^{ \pm}$is on the $b\left(u_{1}\right)$ side of $e$ for $1 \leq j \leq s^{ \pm}$and on the $b\left(u_{2}\right)$ side of $e$ for $s^{ \pm}+1 \leq j \leq r$. Let $d$ be the distance from $b\left(u_{1}\right)$ to $b\left(u_{2}\right)$.

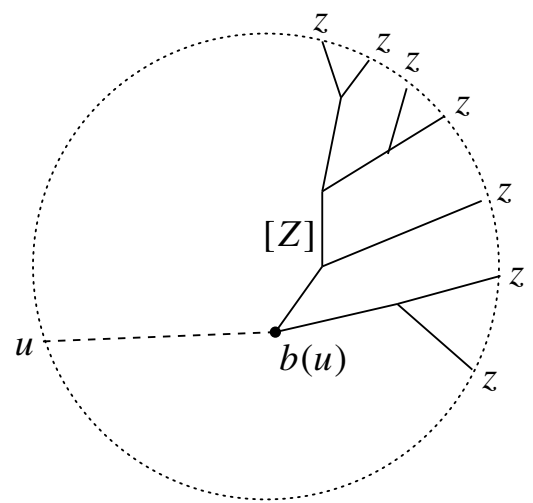

Figure 1. The definition of $b(u)$. 
We have

$$
\begin{aligned}
v\left(\phi_{i}\left(u_{1}\right)\right)-v\left(\phi_{i}\left(u_{2}\right)\right) & =v\left(\frac{\phi_{i}\left(u_{1}\right)}{\phi_{i}\left(u_{2}\right)}\right)=v\left(\frac{\prod_{j=1}^{r}\left(u_{1}-z_{j}^{+}\right) / \prod_{j=1}^{r}\left(u_{1}-z_{j}^{-}\right)}{\prod_{j=1}^{r}\left(u_{2}-z_{j}^{+}\right) / \prod_{j=1}^{r}\left(u_{2}-z_{j}^{-}\right)}\right) \\
& =v\left(\prod_{j=1}^{r} c\left(u_{1}, u_{2}: z_{j}^{+}, z_{j}^{-}\right)\right) \\
& =\sum_{j=1}^{r} v\left(c\left(u_{1}, u_{2}: z_{j}^{+}, z_{j}^{-}\right)\right)=d\left(s^{+}-s^{-}\right) .
\end{aligned}
$$

The last equality is by applying Proposition 4.2 to each term.

By the zero-tension condition, $s_{i}=s^{+}-s^{-}$. So $\iota\left(b\left(u_{1}\right)\right)_{i}-\iota\left(b\left(u_{2}\right)\right)_{i}$ is also $d\left(s^{+}-s^{-}\right)$.

We pause for two examples.

Example 6.3. Consider the tree in $\mathbb{Q}^{3}$ with a finite edge running from $(0,0,0)$ to $(1,1,1)$, infinite edges leaving $(1,1,1)$ in directions $(1,0,0)$ and $(0,1,1)$, and edges departing $(0,0,0)$ in directions $(0,-1,0)$ and $(-1,0,-1)$. Then $\left[0, t, 1, t^{-1}\right]$ is isometric to $\Gamma$, with $0, t, 1$ and $t^{-1}$ respectively corresponding to the endpoints of the above infinite rays. We have

$$
\begin{aligned}
& Z_{1}^{+}=\{0\}, \quad Z_{2}^{+}=\{t\}, \quad Z_{3}^{+}=\{t\}, \\
& Z_{1}^{-}=\left\{t^{-1}\right\}, \quad Z_{2}^{-}=\{1\}, \quad Z_{3}^{-}=\left\{t^{-1}\right\} .
\end{aligned}
$$

Thus, the map $\phi$ is given by

$$
u \mapsto\left(\frac{u}{u-t^{-1}}, \frac{u-t}{u-1}, \frac{u-t}{u-t^{-1}}\right) .
$$

The image of this map is a genus-zero curve $C$ with Trop $C$ equal to the given tree.

Example 6.4. This time we choose a tree with no internal edges but complicated slopes. Consider the tree $T$ in $\mathbb{Q}^{3}$ with no internal edges and four unbounded rays of slope $(1,2,3),(5,-3,4),(-7,1,-2),(1,0,-5)$. Assuming that $\kappa$ has characteristic 0 , the tree $[1,2,3,4] \subset \mathrm{BT}(K)$ is isometric to $T$. Our multisets $Z_{i}^{ \pm}$ are

$$
\begin{array}{lll}
Z_{1}^{+}=\{1,2,2,2,2,2,4\}, & Z_{2}^{+}=\{1,1,3\}, & Z_{3}^{+}=\{1,1,1,2,2,2,2\}, \\
Z_{1}^{-}=\{3,3,3,3,3,3,3\}, & Z_{2}^{-}=\{2,2,2\}, & Z_{3}^{-}=\{3,3,4,4,4,4,4\} .
\end{array}
$$

For example, there are 5 occurrences of the number 4 in $Z_{3}^{-}$because ray number 4 of our tree has slope -5 in the $x_{3}$ direction. 
Our map $\phi$ is given by

$$
u \mapsto\left(\frac{(u-1)(u-2)^{5}(u-4)}{(u-3)^{7}}, \frac{(u-1)^{2}(u-3)}{(u-2)^{3}}, \frac{(u-1)^{3}(u-2)^{4}}{(u-3)^{2}(u-4)^{5}}\right) .
$$

Once again, the image of $\phi$ is a genus-zero curve whose tropicalization is the given tree.

\section{Tropical curves of genus one}

Let $(\Gamma, \iota, m)$ be a zero-tension curve where $\Gamma$ is connected with first Betti number one. This means that $\Gamma$ has a unique cycle; let $e_{1}, \ldots, e_{r}$ be the edges of this cycle and let $\sigma_{i}$ be the slope $\sigma\left(e_{i}\right)$. Recall that $\sigma\left(e_{i}\right)=m\left(e_{i}\right) \rho\left(e_{i}\right)$, where $\rho$ is the minimal lattice vector along $e_{i}$ and $m$ is the multiplicity of edge $e_{i}$.

Our aim in this section is to prove:

Theorem 3.3. Let $(\iota, \Gamma, m)$ be a zero-tension curve of genus one and degree $\delta$. Assume that the slopes $\sigma_{1}, \sigma_{2}, \ldots, \sigma_{r}$ span $\mathbb{Q} \otimes N$. Then there is a (punctured) genus-one curve $C$ over $\mathbb{K}$ of degree $\delta$ and a map $\phi: C \rightarrow \mathbb{T}(\mathbb{K}, N)$ such that $\iota(\Gamma)=\operatorname{Trop} \phi(C)$.

We use Tate's nonarchimedean uniformizations of elliptic curves. A good reference for this subject is Sections 2 and 3 of [Roquette 1970]. Let $q \in \mathbb{K}^{*}$ with $v(q)>0$. Tate constructs an elliptic curve $E$ over $\mathbb{K}$ with a bijection $\mathscr{P}$ from $\mathbb{K}^{*} / q^{\mathbb{Z}}$ to $E(\mathbb{K})$. For $u$ and $z \in \mathbb{K}^{*}$, define

$$
\Theta(u)=\prod_{j=-\infty}^{0}\left(1-q^{-j} u\right) \prod_{j=1}^{\infty}\left(1-q^{j} / u\right) .
$$

For any $u$ and $q$ in $\mathbb{K}^{*}$, there is a finite extension $K$ of $\boldsymbol{K}$ containing $u$ and $q$ and this product is convergent in the nonarchimedean topology on $K$ as long as none of the individual terms are zero. Thus, the product above is well defined for all $u \in \mathbb{K}^{*} \backslash q^{\mathbb{Z}}$ and it satisfies $\Theta(q u)=(-1 / u) \Theta(u)$. (Remember that $\lim _{n \rightarrow \infty} q^{n}=0$ because $v(q)>0$.) Thus, if $Z^{+}=\left\{z_{1}^{+}, \ldots, z_{k}^{+}\right\}$and $Z^{-}=\left\{z_{1}^{-}, \ldots, z_{k}^{-}\right\}$are finite multisubsets of $K^{*}$ with the same cardinality and $\prod_{i=1}^{k} z_{i}^{+}=\prod_{i=1}^{k} z_{i}^{-}$, then

$$
\phi\left(u ; Z_{i}^{+}, Z_{i}^{-}\right):=\prod_{i=1}^{k} \frac{\Theta\left(u / z_{i}^{+}\right)}{\Theta\left(u / z_{i}^{-}\right)}
$$

is a well-defined function on $\left(\mathbb{K}^{*} / q^{\mathbb{Z}}\right) \backslash\left(\bigcup_{j=\infty}^{\infty} q^{j} \cdot\left\{z_{1}^{+}, \ldots, z_{k}^{+}, z_{1}^{-}, \ldots, z_{k}^{-}\right\}\right)$. Consider this product as a function on $E(\mathbb{K})$ with the appropriate points removed. Tate proves that this is a meromorphic function of $u$ with zeroes at the points $\mathscr{P}\left(z_{i}^{+}\right)$ and poles at $\mathscr{P}\left(z_{i}^{-}\right)$. Every nonzero meromorphic function on $E(\mathbb{K})$, up to scalar multiples, occurs in this way. (See [Roquette 1970, Section 2, Proposition 1] for 
the statement that all the nonzero functions in the field which Roquette calls $M_{K}$ are of this form. See [Roquette 1970, Section 2, Statement IV] for the fact that this field is the meromorphic functions on an elliptic curve over $\mathbb{K}$.)

Remark. By the Jacobi triple product formula, $\Theta(u)$ can be given by the alternate formula $\prod_{i=0}^{\infty}\left(1-q^{i}\right)^{-1} \times \sum_{n=-\infty}^{\infty} q^{\left(\begin{array}{c}n \\ 2\end{array}\right)}(-u)^{n}$. But this formula will not be useful to us.

Our goal, given the input data $(\iota, \Gamma, m)$, is to construct a genus-one curve $C$ over $\mathbb{K}$ and a rational map $\phi: C \rightarrow\left(\mathbb{K}^{*}\right)^{n}$ such that $\operatorname{Trop} \phi(C)=\iota(\Gamma)$. The way our construction will proceed is to use $(\iota, \Gamma, m)$ to construct an element $q \in \mathbb{K}^{*}$, with $v(q)>0$, and finite multisubsets $Z_{1}^{+}, \ldots, Z_{n}^{+}, Z_{1}^{-}, \ldots, Z_{n}^{-}$, of $\mathbb{P}^{1}(\mathbb{K})$. We will have $\prod_{z \in Z_{i}^{+}} z=\prod_{z \in Z_{i}^{-}}$, which will be the hardest part of the construction to achieve. Thus, we will have a rational map $\phi$ from the Tate curve $C:=\mathbb{K}^{*} / q^{\mathbb{Z}}$ to $\left(\mathbb{K}^{*}\right)^{n}$ by $\mathscr{P}(u) \mapsto\left(\phi\left(u ; Z_{1}^{+}, Z_{1}^{-}\right), \ldots, \phi\left(u ; Z_{n}^{+}, Z_{n}^{-}\right)\right)$. We will have arranged our choices so that $\operatorname{Trop} \phi(C)=\iota(\Gamma)$. We often abbreviate $\phi\left(u ; Z_{i}^{+}, Z_{i}^{-}\right)$to $\phi_{i}(u)$, when the choice of $Z_{i}^{ \pm}$is clear.

We begin by discussing the situation for an arbitrary choice of $q$ and $Z_{1}^{ \pm}, \ldots, Z_{n}^{ \pm}$. Later, we will specialize our discussion to the particular choices that will derive from the graph $\Gamma$. We will use $I\left(q, Z_{\bullet}^{ \pm}\right)$to denote the graph we will construct from $q$ and $Z_{1}^{ \pm}, \ldots, Z_{n}^{ \pm}$; eventually $\beth$ will be isomorphic to $\Gamma{ }^{8}$ We will drop the arguments of $I$ when they should be free from context.

So, let $q \in \mathbb{K}^{*}$ with $v(q)>0$ and let $Z_{1}^{+}, Z_{1}^{-}, \ldots, Z_{n}^{+}, Z_{n}^{-}$be $2 n$ finite nonempty multisubsets of $\mathbb{K}^{*}$ with $\left|Z_{i}^{+}\right|=\left|Z_{i}^{-}\right|$for $i=1,2, \ldots, n$. We introduce the notation $Z$ for

$$
\bigcup_{i=-\infty}^{\infty} \bigcup_{j=1}^{n} q^{i}\left(Z_{j}^{+} \cup Z_{j}^{-}\right) \text {. }
$$

Let $^{9} \tilde{\beth}\left(q, Z_{\bullet}^{ \pm}\right)=\bigcup_{z, z^{\prime} \in Z}\left[z, z^{\prime}\right] \subset \mathrm{BT}(\mathbb{K})$. The metric space $\tilde{\Xi}\left(q, Z_{\bullet}^{ \pm}\right)$is invariant under multiplication by $q^{\mathbb{Z}}$; we define $\beth\left(q, Z_{\bullet}^{ \pm}\right)=\tilde{\Xi}\left(q, Z_{\bullet}^{ \pm}\right) / q^{\mathbb{Z}}$.

Lemma 7.1. The metric space $\tilde{\Xi}\left(q, Z_{\bullet}^{ \pm}\right)$is an infinite tree. The quotient $\tilde{\beth}\left(q, Z_{\bullet}^{ \pm}\right) / q^{\mathbb{Z}}$ is a finite graph with first Betti number one, and $\Xi\left(q, Z_{\bullet}^{ \pm}\right)$is isometric to a dense subset of $U$.

Note that we call a graph "finite" when it has a finite number of vertices and of edges. When the graph is equipped with a metric, as $\tilde{I}$ is, we do not take "finite" to imply that all of the edges have finite length.

Proof. First note that for any $a$ and $b \in \mathbb{K}^{*}$ and any $d \in[0, \infty]$, we either have $d \in\left[a, q^{j} b\right]$ for $j$ sufficiently large or we have $d \in\left[a, q^{-j} b\right]$ for $j$ sufficiently large.

${ }^{8}$ The symbol I is the Hebrew letter "gimmel", which makes the same sound as the Greek letter $\Gamma$.

${ }^{9}$ We will systematically use the tilde, $\sim$, for objects associated to the universal cover. 

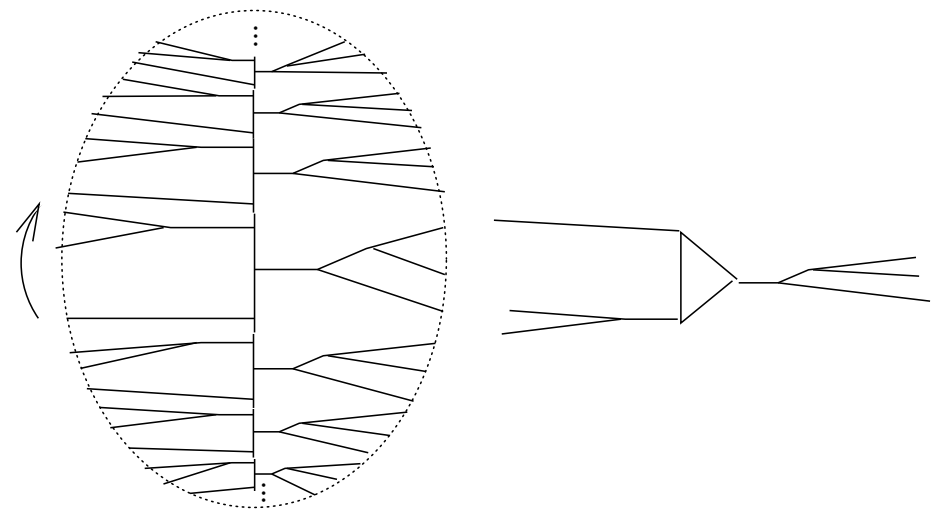

Figure 2. The graphs $\tilde{\beth}$ and $\beth$.

The same holds if we take $d \in[a, \infty]$ or $d \in[a, 0]$. So $\tilde{\bar{I}}$, which by definition is $\bigcup_{z, z^{\prime} \in Z}\left[z, z^{\prime}\right]$, is also $\bigcup_{z, z^{\prime} \in Z}\left[0, \infty, z, z^{\prime}\right]$. Also, note that for any $a$ and $b \in \mathbb{K}^{*}$, we have $[a, b, 0, \infty]=[a, 0, \infty] \cup[b, 0, \infty]$. (Just check the three possible topologies for the tree $[a, b, 0, \infty]$.) So $\tilde{\beth}=\bigcup_{z \in Z}[0, z, \infty]$.

For $u \in \mathbb{Q}$, set $T_{u}=\bigcup_{z \in Z, v(z)=u}[0, z, \infty]$, so $\tilde{J}=\bigcup_{u \in \mathbb{Q}} T_{u}$. If $u \neq u^{\prime}$ then $T_{u} \cap T_{u^{\prime}}=[0, \infty]$. So $\tilde{\beth}$ is just a central path $[0, \infty]$ with the side stalk $T_{u} \backslash[0, \infty]$ stuck on for each $u \in v(Z)$. But, for every $u$, there are only finitely many $u \in Z$ with valuation $u$, so $T_{u}$ is just a finite tree. And $v(Z)$ is a union of finitely many copies of $v(q) \cdot \mathbb{Z}$, so in particular $v(Z)$ is a discrete subset of $A$. So we see that $\tilde{\beth}$ really is just an infinite tree, consisting of an infinite path with a periodic pattern of finite trees branching off from it.

When we take the quotient of this infinite tree by the periodic shift by $v(q)$ along this path, we get a finite graph.

In Figure 2, we show $\tilde{\beth}$ on the left and I on the right for a sample choice of $q$ and $Z$. The action of $q$ is shown by the boldfaced arrow.

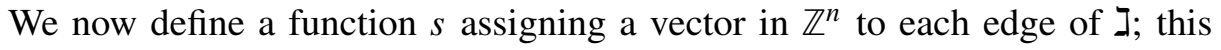
function will eventually describe the slopes of edges of our tropical curve. Fix an index $i$ between 1 and $n$ and an oriented edge $\tilde{e}$ of $\tilde{\beth}$. Removing $\tilde{e}$ from $\tilde{I}$ divides $\tilde{I}$ into two components. This gives rise to partitions $Z_{i}^{ \pm}=H_{i}^{ \pm} \sqcup T_{i}^{ \pm}$of $Z_{i}^{ \pm}$, where $H_{i}^{ \pm}$consists of those elements of $Z_{i}^{ \pm}$which are on the "head" side of $\tilde{e}$ and $T_{i}^{ \pm}$ consists of elements of $Z_{i}^{ \pm}$which are on the "tail" side of $\tilde{e}$. Define

$$
\tilde{s}_{i}(\tilde{e})=\left|H_{i}^{+}\right|-\left|T_{i}^{+}\right|-\left|H_{i}^{-}\right|+\left|T_{i}^{-}\right| .
$$

Note that each of these multisets is finite, so $\tilde{s}_{i}(\tilde{e})$ is well defined. Also, note that $\tilde{s}_{i}(\tilde{e})$ is zero for all but finitely many $e$. We now define

$$
s_{i}(e)=\sum_{\tilde{e} \text { lifts } e} \tilde{s}_{i}(\tilde{e}) .
$$


Here we are summing over all the edges $\tilde{e}$ of $\tilde{\beth}$ above the edge $e$ of $\beth$. Note that all but finitely many terms of this sum are zero. We then define $s(e)$ to be the vector $\left(s_{1}(e), \ldots, s_{n}(e)\right)$. Note that $s(e)=s(q e)$, so we may think of $s$ a function on directed edges of $\beth$, and note that reversing an edge negates $s$.

Lemma 7.2. The vectors $s(e)$ obey the zero-tension condition.

Proof. We will show that the function $\tilde{s}$ on the edges of $\tilde{\bar{J}}$ obeys the zero-tension condition, so the translates of $\tilde{s}$ will as well, and hence their sum $s$ will. Let $v$ be an internal vertex of $\tilde{\beth}$ and let $e_{1}, e_{2}, \ldots, e_{p}$ be the edges incident to $v$, which we will direct away from $v$. Fix an index $i$ between 1 and $n$; we need to show that $\sum_{k} \tilde{s}\left(e_{k}\right)_{i}=0$. Let $T_{k}$ be the component of $\tilde{\mathcal{J}} \backslash\{v\}$ which contains the interior of $e_{k}$. Let $D_{k}$ be the difference between the cardinalities of $Z_{i}^{+} \cap T_{k}$ and $Z_{i}^{-} \cap T_{k}$. Since $\left|Z_{i}^{+}\right|=\left|Z_{i}^{-}\right|$, we know that $\sum D_{k}=0$. We have $\tilde{s}\left(e_{k}\right)_{i}=D_{k}-\sum_{m \neq k} D_{m}$, so $\sum_{k=1}^{p} \tilde{s}\left(e_{k}\right)_{i}=(p-1) \sum_{k=1}^{p} D_{k}=0$.

We will now consider the effect of adding the following additional condition:

$$
\text { for } i=1, \ldots, n, \quad v\left(\prod_{z \in Z_{i}^{+}} z\right)=v\left(\prod_{z \in Z_{i}^{-}} z\right) .
$$

Note that condition (1) is an immediate consequence of the stronger condition

$$
\text { for } i=1, \ldots, n, \quad \prod_{z \in Z_{i}^{+}} z=\prod_{z \in Z_{i}^{-}} z .
$$

Proposition 7.3. Suppose that condition (1) holds. Let $e_{1}, e_{2}, \ldots, e_{r}$ be the edges of the unique cycle of $\beth$, ordered and oriented cyclically. Let $\ell(e)$ be the length of the edge e of $\tilde{\beth}$. Then

$$
\sum_{k=1}^{r} \ell\left(e_{k}\right) s\left(e_{k}\right)=0
$$

Proof. Pick an index $i$ between 1 and $n$; we will show that $\sum_{k=1}^{r} \ell\left(e_{k}\right) s_{i}\left(e_{k}\right)=0$. Recall that we have $\left|Z_{i}^{+}\right|=\left|Z_{i}^{-}\right|$. Set $N=\left|Z_{i}^{+}\right|=\left|Z_{i}^{-}\right|$and fix orderings $\left(z_{1}^{ \pm}, z_{2}^{ \pm}, \ldots, z_{N}^{ \pm}\right)$of $Z_{i}^{+}$and $Z_{i}^{-}$. For an oriented edge $e$ of $\tilde{\beth}$ and an index $m$ between 1 and $N$, define $\delta_{m}(e)$ to be 1 if $z_{m}^{+}$is on the head side of $e$ and $z_{m}^{-}$is on the tail side of e; define $\delta_{m}(e)$ to be -1 if $z_{m}^{+}$is on the tail side of $e$ and $z_{m}^{-}$is on the head side of e; define $\delta_{m}(e)$ to be 0 if $z_{m}^{+}$and $z_{m}^{-}$are on the same side of $e$. Then $\tilde{s}_{i}(e)=\sum_{m=1}^{N} \delta_{m}(e)$.

Now we have

$$
\sum_{k=1}^{r} \ell\left(e_{k}\right) s_{i}\left(e_{k}\right)=\sum_{k=1}^{r} \ell\left(e_{k}\right) \sum_{j=-\infty}^{\infty} \tilde{s}_{i}\left(q^{j} e_{k}\right)=\sum_{e \subset[0, \infty]} \ell(e) \tilde{s}_{i}(e) .
$$


Using the expression in the previous paragraph for $\tilde{s}_{i}(e)$, we see that this equals

$$
\sum_{e \subset[0, \infty]} \ell(e) \sum_{m=1}^{N} \delta_{m}(e)=\sum_{m=1}^{N} \sum_{e \subset[0, \infty]} \ell(e) \delta_{m}(e),
$$

where we may interchange summation because all but finitely many terms are zero. Now the inner sum $\sum_{e \subset[0, \infty]} \ell(e) \delta_{m}(e)$ is the (signed) sum of the lengths of all edges in $[0, \infty]$ which separate $z_{m}^{+}$from $z_{m}^{-}$. In other words, the inner sum is the signed length of $[0, \infty] \cap\left[z_{m}^{+}, z_{m}^{-}\right]$. But, by Proposition 4.2, this is simply $v\left(z_{m}^{+}\right)-v\left(z_{m}^{-}\right)$. Plugging this into our sum, we see that the quantity we wish to show is zero is $\sum_{m=1}^{N}\left(v\left(z_{m}^{+}\right)-v\left(z_{m}^{-}\right)\right)$. But, by condition (1), we have $v\left(\prod_{z \in Z_{i}^{+}} z\right)-v\left(\prod_{z \in Z_{i}^{-}} z\right)=0$, so $\sum_{m=1}^{N}\left(v\left(z_{m}^{+}\right)-v\left(z_{m}^{-}\right)\right)=0$.

We now build a continuous map $\tilde{f}: \tilde{\Xi} \rightarrow \mathbb{Q}^{n}$. If $e$ is any finite edge of $\tilde{\beth}$, directed from vertex $x$ to vertex $y$, then $\tilde{f}(y)=\tilde{f}(x)+\ell(e) s(e)$ and $\tilde{f}(e)$ is the line segment connecting $\tilde{f}(x)$ and $\tilde{f}(y)$. If $e$ is an infinite ray of $\beth$ then $\tilde{f}(e)$ is an unbounded ray of slope $s(e)$. This map is determined by the $s(e)$ up to translation by an element of $\mathbb{Q}^{n}$. Using Proposition 7.3, we see that $\tilde{f}$ factors through the quotient graph $\mathrm{I}$; we will write the map $\beth \rightarrow \mathbb{Q}^{n}$ as $f$. We now come to the fundamental computation that unites our combinatorial constructions with actual geometry:

Proposition 7.4. Given $q$ and $Z_{1}^{ \pm}, \ldots, Z_{n}^{ \pm}$subject to condition (2), define the curve $C$ and the map $\phi$ as described at the beginning of this section. Define also the graph I and the map $f$. (Since we have assumed (2), we have (1), so we can define $f$.) Then Trop $\phi(C)$ is a translation of $f(\beth)$ and they have the same degree.

This proof is very similar to the proof of Theorem 6.2. We will make the inconsequential abuse of notation of considering $\phi$ both as a map from (an open subset of) $C$ and from $\mathbb{K}^{*} \backslash Z$. Here $Z$, as above, is $\bigcup_{i=\infty}^{\infty} \bigcup_{j=1}^{n} q^{i}\left(Z_{j}^{+} \cup Z_{j}^{-}\right)$.

Proof. Let $u \in \mathbb{K}^{*} \backslash Z$. Then $[Z \cup\{u\}]$ is a tree, which is the union of $\tilde{\Xi}$ and a path ending at $u$. Let $b(u) \in \tilde{\Xi}$ be the other end of this path. We claim that, up to a translation, $v(\phi(u))$ is $f(b(u))$. In other words, if $u_{1}$ and $u_{2}$ are distinct members of $u \in \mathbb{K}^{*} \backslash Z$, we must show that for each $i$ between 1 and $n$, we have

$$
v\left(\phi_{i}\left(u_{1}\right)\right)-v\left(\phi_{i}\left(u_{2}\right)\right)=f\left(b\left(u_{1}\right)\right)_{i}-f\left(b\left(u_{2}\right)\right)_{i} .
$$

It is enough to show this in the case where $b\left(u_{1}\right)$ and $b\left(u_{2}\right)$ lie in the same edge $e$ of $[Z]$. Let $s(e)=\left(s_{1}, \ldots, s_{n}\right)$. We will fix one coordinate $i$ to pay attention to, so $i$ will not appear in our notation. Let $Z_{i}^{+}=\left\{z_{1}^{+}, \ldots, z_{N}^{+}\right\}$and $Z_{i}^{-}=\left\{z_{1}^{-}, \ldots, z_{N}^{-}\right\}$. Let $d$ be the distance from $b\left(u_{1}\right)$ to $b\left(u_{2}\right)$. 
We have

$$
\begin{aligned}
v\left(\phi_{i}\left(u_{1}\right)\right)-v\left(\phi_{i}\left(u_{2}\right)\right) & =v\left(\frac{\phi_{i}\left(u_{1}\right)}{\phi_{i}\left(u_{2}\right)}\right)=v\left(\frac{\prod_{m=1}^{N} \Theta\left(u_{1} / z_{m}^{+}\right) / \Theta\left(u_{1} / z_{m}^{-}\right)}{\prod_{m=1}^{N} \Theta\left(u_{2} / z_{m}^{+}\right) / \Theta\left(u_{2} / z_{m}^{-}\right)}\right) \\
& =\sum_{m=1}^{N} v\left(\frac{\Theta\left(u_{1} / z_{m}^{+}\right) / \Theta\left(u_{1} / z_{m}^{-}\right)}{\Theta\left(u_{2} / z_{m}^{+}\right) / \Theta\left(u_{2} / z_{m}^{-}\right)}\right) .
\end{aligned}
$$

Now, by definition,

$$
\begin{aligned}
& \frac{\Theta\left(u_{1} / z_{m}^{+}\right) / \Theta\left(u_{1} / z_{m}^{-}\right)}{\Theta\left(u_{2} / z_{m}^{+}\right) / \Theta\left(u_{2} / z_{m}^{-}\right)}= \prod_{j=-\infty}^{0}\left(\frac{\left(1-q^{-j} u_{1} / z_{m}^{+}\right)\left(1-q^{-j} u_{2} / z_{m}^{-}\right)}{\left(1-q^{-j} u_{1} / z_{m}^{-}\right)\left(1-q^{-j} u_{2} / z_{m}^{+}\right)}\right) \\
& \quad \times \prod_{j=1}^{\infty}\left(\frac{\left(1-q^{j} z_{m}^{+} / u_{1}\right)\left(1-q^{j} z_{m}^{-} / u_{2}\right)}{\left(1-q^{j} z_{m}^{-} / u_{1}\right)\left(1-q^{j} z_{m}^{+} / u_{2}\right)}\right) \\
&=\prod_{j=-\infty}^{\infty} c\left(q^{-j} u_{1}, q^{-j} u_{2}: z_{m}^{-}, z_{m}^{-}\right) .
\end{aligned}
$$

Here we may rearrange our product freely because in nonarchimedean analysis all convergent sums and products converge absolutely. So, by Proposition 4.2, we can compute the valuation of (4):

$$
v\left(\frac{\Theta\left(u_{1} / z_{m}^{+}\right) / \Theta\left(u_{1} / z_{m}^{-}\right)}{\Theta\left(u_{2} / z_{m}^{+}\right) / \Theta\left(u_{2} / z_{m}^{-}\right)}\right)=\sum_{j=-\infty}^{\infty}\left(\text { signed length of }\left[u_{1} / q^{j}, u_{2} / q^{j}\right] \cap\left[z_{m}^{+}, z_{m}^{-}\right]\right) \text {. }
$$

Now, the sum on $m$ of the signed length of $\left[u_{1} / q^{j}, u_{2} / q^{j}\right] \cap\left[z_{m}^{+}, z_{m}^{-}\right]$is $d \tilde{s}_{i}\left(q^{j} e\right)$. So, plugging (5) into (3) and interchanging summation, we obtain

$$
v\left(\phi_{i}\left(u_{1}\right)\right)-v\left(\phi_{i}\left(u_{2}\right)\right)=\sum_{j=-\infty}^{\infty} d \tilde{s}_{i}\left(q^{j} e\right)=d s_{i}(e)=f\left(b\left(u_{1}\right)\right)_{i}-f\left(b\left(u_{2}\right)\right)_{i} .
$$

This is the desired formula.

We now know how to build a genus-one curve over $\mathbb{K}$ with a rational map $\phi: C \rightarrow\left(\mathbb{K}^{*}\right)^{n}$ such that Trop $\phi(C)$ has a given form. In order to prove Theorem 3.3, we need to use $(\iota, \Gamma, m)$ to find $q$ and to find multisubsets $Z_{1}^{ \pm}, \ldots, Z_{n}^{ \pm}$of $\mathbb{K}^{*}$ such that

(i) for $i=1, \ldots, n$, we have $\left|Z_{i}^{+}\right|=\left|Z_{i}^{-}\right|$;

(ii) for $i=1, \ldots, n$, we have $\prod_{z \in Z_{i}^{+}} z=\prod_{z \in Z_{i}^{-}} z$;

(iii) the graph $\beth\left(q, Z_{\bullet}^{ \pm}\right)$is isometric to $\Gamma$; and

(iv) the map $f$ corresponds to $\iota$ under the isometry $\exists\left(q, Z_{\bullet}^{ \pm}\right) \cong \Gamma$. 
We spend the rest of this section describing the necessary construction. We will first build sets $Y_{1}^{ \pm}, \ldots, Y_{n}^{ \pm}$which obey all of these conditions except that, instead of condition (ii), they satisfy the weaker (1) (stated before Proposition 7.3). We will then perturb the $Y_{i}^{ \pm}$to give sets $Z_{i}^{ \pm}$satisfying this last condition.

Let $\ell$ be the length of the circuit of $\Gamma$. First, we choose ${ }^{10} q$ so that $v(q)=\ell$. Let $\tilde{\Gamma}$ be the universal cover of $\Gamma$. The graph $\tilde{\Gamma}$ is an infinite tree which has one doubly infinite path with finite trees periodically branching off this path. Choose a point in the interior of an edge $e_{0}$ of the circuit of $\Gamma$ and cut $\Gamma$ at this point to produce a tree $T$, so $\tilde{\Gamma}$ is a union of a doubly infinite sequence of copies of $T$. The tree $T$ has two finite rays and a number of infinite rays. By Proposition 6.1, we can find a subset $U$ of $\overline{\mathrm{BT}}(\mathbb{K})$ such that $T \cong[U]$. There are two elements of $\mathrm{BT}(\mathbb{K})$ in $U$, which we will call $u_{0}$ and $u_{1}$; the rest of $U$ is contained in $\mathbb{P}^{1}(\mathbb{K})$. The distance from $u_{0}$ to $u_{1}$ is $\ell=v(q)$, so we may (and do) use a transformation in $\mathrm{GL}_{2}(\mathbb{K})$ to assume that $u_{0}=\operatorname{Span}_{\overparen{O}}((1,0),(0,1))$ and $u_{1}=\operatorname{Span}_{\mathscr{O}}((q, 0),(0,1))$. Since $u_{0}$ and $u_{1}$ are leaves of [U], all the other elements of $U \backslash\left\{u_{0}, u_{1}\right\}$ must be contained in $v^{-1}((0, \ell)) \subset \mathbb{K}^{*} \subset \mathbb{P}^{1}(\mathbb{K})$. We set $Y=\bigcup_{j=-\infty}^{\infty} q^{j}\left(U \backslash\left\{u_{0}, u_{1}\right\}\right)$. Then $[Y] \cong \tilde{\Gamma}$.

We now describe how to choose the multisets $Y_{1}^{ \pm}, \ldots, Y_{n}^{ \pm}$as subsets of the set $Y$. Fix an index $i$ between 1 and $n$. We first define multisubsets $W_{i}^{+}$and $W_{i}^{-}$of $U$. Let $v$ be a leaf of $\Gamma$, at the end of the edge $e$, and let $u$ be the element of $U$ at the end of the corresponding ray of $T$. Then $u$ will occur $\left|\sigma_{v}(e)_{i}\right|$ times in $W_{i}^{ \pm}$, where \pm is the opposite of the sign of $\sigma_{v}(e)_{i}$, and will not occur at all in $W_{i}^{\mp}$. Note that the assumption that $(\iota, \Gamma, m)$ is zero-tension forces $W_{i}^{+}$and $W_{i}^{-}$to have the same cardinality. We now modify $W_{i}^{ \pm}$slightly to produce $Y_{i}^{ \pm}$. Let $\left(s_{1}, \ldots, s_{n}\right)$ be the slope of the edge $e_{0}$ which we cut to make $T$. For each $i$ between 1 and $n$, we take one element of $W_{i}^{+}$and multiply it by $q^{s_{i}}$, leaving the other elements of $W_{i}^{+}$unchanged. We call the resulting multiset $Y_{i}^{+}$. We take $Y_{i}^{-}=W_{i}^{-}$. Then $\bigcup_{j=-\infty}^{\infty} q^{j} \bigcup_{i=1}^{n}\left(Y_{i}^{+} \cup Y_{i}^{-}\right)=Y$, so we have $\tilde{\Xi}\left(q, Y_{\bullet}^{ \pm}\right) \cong[Y] \cong \tilde{\Gamma}$ and $\Xi\left(q, Y_{\bullet}^{ \pm}\right) \cong \Gamma$.

We need to check that if $e$ is an oriented edge of $\Gamma$, then $\sigma(e)$ is equal to $s(e)$. (Note that when writing $s(e)$, we have thought of $e$ as an edge of $\beth$.) The $s(e)$ obey the zero-tension condition, by Lemma 7.2, so it is enough to check that $s(e)=\sigma(e)$ for (1) $e$ an unbounded ray and (2) $e=e_{0}$; the zero-tension condition will then determine both $s(e)$ and $\sigma(e)$ for the remaining edges. If $e$ is an unbounded ray, with the leaf $v$ at its end, then we forced there to be $\mp \sigma_{v}(e)_{i}$ elements of $Y_{i}^{ \pm}$lying at the end of the preimages of $e$ in $\tilde{\bar{I}}$. For each unbounded ray $e^{\prime}$ of $\tilde{\bar{J}}$ (directed towards its leaf), $\tilde{s}\left(e^{\prime}\right)_{i}$ is \pm times the number of elements of $Y_{i}^{ \pm}$at the end of $e^{\prime}$. So $\sigma(e)_{i}$ is the sum of $\tilde{s}\left(e^{\prime}\right)_{i}$ over all preimages $e^{\prime}$ of $e$. This is precisely the definition of $s(e)_{i}$.

${ }^{10}$ This construction works for any $q$ of the correct valuation. This is probably unfortunate; it suggests that these methods will need further improvement before they can be used for enumerative problems which are only solvable for certain particular $j$-invariants. 
Now let us consider the case $e=e_{0}$. If we computed $s\left(e_{0}\right)_{i}$ using $Y_{i}^{+}$and $Y_{i}^{-}$, we would get zero, as for every preimage $e^{\prime}$ of $e$ in $\tilde{\bar{I}}$, all of $U \backslash\left\{u_{0}, u_{1}\right\}$ lies to one side of $e^{\prime}$. When we use $Y_{i}^{+}$and $Y_{i}^{-}$instead, only one element moves and that element moves past $\sigma\left(e_{0}\right)_{i}$ preimages of $e_{0}$, and we have $\sigma\left(e_{0}\right)_{i}=s\left(e_{0}\right)_{i}$.

Lemma 7.5. For each $i$ between 1 and $n$, we have $v\left(\prod_{z \in Y_{i}^{+}} z\right)=v\left(\prod_{z \in Y_{i}^{-}} z\right)$.

This proof is essentially reversing the proof of Proposition 7.3.

Proof. Fix an index $i$ between 1 and $n$. We choose an ordering $\left(y_{1}^{+}, y_{2}^{+}, \ldots, y_{m}^{+}\right)$ of $Y_{i}^{+}$and an ordering $\left(y_{1}^{-}, y_{2}^{-}, \ldots, y_{m}^{-}\right)$of $Y_{i}^{-}$. We want to establish that

$$
\sum_{k=1}^{m} v\left(y_{k}^{+} / y_{k}^{-}\right)=0 .
$$

Now, $y_{k}^{+} / y_{k}^{-}$is the cross ratio $c\left(y_{k}^{+}, y_{k}^{-}: 0, \infty\right)$, so by Proposition $4.2, v\left(y_{k}^{+} / y_{k}^{-}\right)$is the signed length of the intersection of $[0, \infty]$ with $\left[y_{k}^{+}, y_{k}^{-}\right]$. We can break up this intersection as a sum over the various edges in the doubly infinite path $[0, \infty]$, so $v\left(y_{k}^{+} / y_{k}^{-}\right)= \pm \sum_{e^{\prime} \in[0, \infty] \cap\left[y_{k}^{+}, y_{k}^{-}\right]} \ell\left(e^{\prime}\right)$, where $\ell\left(e^{\prime}\right)$ is the length of $e^{\prime}$ and the sign describes whether or not the orientation of the path $\left[y_{k}^{+}, y_{k}^{-}\right]$matches the orientation of $e^{\prime}$ from 0 to $\infty$.

We know that for any edge $e_{j}$ in the circuit of $\Gamma$, the signed number of intersections of the paths $\left[y_{1}^{+}, y_{1}^{-}\right], \ldots,\left[y_{m}^{+}, y_{m}^{-}\right]$with the preimages of $e_{j}$ is $s\left(e_{j}\right)_{i}$. So $\sum_{k=1}^{m} v\left(y_{k}^{+} / y_{k}^{-}\right)=\sum_{j=1}^{r} \ell\left(e_{j}\right) s\left(e_{j}\right)_{i}$. But the $j$-th summand on the right is the displacement in the $i$-th coordinate between the two endpoints of $\iota\left(e_{j}\right)$. Since the edges $\iota\left(e_{1}\right), \iota\left(e_{2}\right), \ldots, \iota\left(e_{r}\right)$ form a closed loop, the sum telescopes to zero.

Now that we have proven Lemma 7.5, we can speak of the map $f$.

We have now shown that $\beth \cong \Gamma$, and that, under this identification, $\sigma=s$. It follows that $f(\beth)$ is a translation of $\iota(\Gamma)$, and we will no longer distinguish $\beth$ from $\Gamma$. If we had $\prod_{z \in Y_{i}^{+}} z=\prod_{z \in Y_{i}^{-}} z$, so that the map $\phi: C \rightarrow\left(\mathbb{K}^{*}\right)^{n}$ would exist, then Trop $\phi(C)$ would be a translation of $f(\Gamma)$. So we will have established Theorem 3.3 if we can simply show that $\prod_{z \in Y_{i}^{+}} z=\prod_{z \in Y_{i}^{-}} z$. More precisely, what we show is that we can perturb each $Y_{i}^{ \pm}$to $Z_{i}^{ \pm}$, so that this product relation holds, without altering $\tilde{\beth}$ as an abstract tree.

We now describe our perturbative method. We remember that the edges of the circuit of $\Gamma$ are called $e_{1}, \ldots, e_{r}$ and we introduce the notation $\left(v_{j-1}, v_{j}\right)$ for the endpoints of $e_{j}$, where the indices are cyclic modulo $r$. Delete the interiors of the edges $e_{1}, \ldots, e_{r}$ from $\Gamma$. Let $T_{j}$ be the component of the remaining forest containing the vertex $v_{j}$ of $\Gamma$. We will choose constants $u_{1}, \ldots, u_{r}$ in $\mathbb{K}^{*}$ with $v\left(u_{1}\right)=\cdots=v\left(u_{r}\right)=0$, which we think of as associated to the $T_{j}$. We modify $Y_{i}^{ \pm}$as follows: Let $y$ be an element of $Y_{i}^{ \pm}$. Consider the component $T_{j}$ to which 
$y$ is attached. Multiply $y$ by $u_{j}$ to obtain a new element $z$. The multiset of thus modified elements will form the set $Z_{i}^{ \pm}$.

Now, multiplication by an element $u$ of $\mathbb{K}^{*}$ with $v(u)=0$ is an automorphism of BT $(\mathbb{K})$ which fixes (pointwise) the path $[0, \infty]$. This transformation modifies each component of $\tilde{\bar{I}} \backslash[0, \infty]$ by such an automorphism, so $\tilde{\bar{I}}$ is left unchanged as an abstract tree. So we must understand how multiplication by $u_{j}$ affects the ratio $\prod_{z \in Z_{i}^{+}} z / \prod_{z \in Z_{i}^{-}} z$. All of the elements of $Y_{i}^{ \pm}$which are in $T_{j}$ are multiplied by $u_{j}$. Let $a_{i j}=\left|Y_{i}^{+} \cap T_{j}\right|-\left|Y_{i}^{-} \cap T_{j}\right|$. So our modification of the $Y^{ \pm}$multiplies $\prod_{z \in Y_{i}^{+}} z / \prod_{z \in Y_{i}^{-}} z$ by $\prod_{j=1}^{r} u_{j}^{a_{i j}}$.

We want to know that we can choose $u_{1}, \ldots, u_{r}$ in $v^{-1}(0)$ such that $\prod_{j=1}^{r} u_{j}^{a_{i j}}=$ $\prod_{z \in Y_{i}^{+}} z / \prod_{z \in Y_{i}^{-}} z$ for $i=1, \ldots, n$. We have shown (Lemma 7.5) that the right-hand side of this equation is in $v^{-1}(0)$. So we want to know that the map of abelian groups $v^{-1}(0)^{r} \mapsto v^{-1}(0)^{n}$ given by the matrix $A:=\left(a_{i j}\right)$ is surjective. Since $\mathbb{K}$ is algebraically closed, $v^{-1}(0)$ is a divisible group and hence it is enough to know that $A$ has rank $n$ over $\mathbb{Q}$. We now turn to verifying this.

Let $\Gamma^{\prime}$ be the graph obtained by taking the circuit of $\Gamma$ and adding an unbounded ray $r_{j}$ at each vertex $v_{j}$. Let $\iota^{\prime}$ be the map from $\Gamma^{\prime}$ into $\mathbb{Q}^{n}$, where $\iota^{\prime}$ restricted to the circuit of $\Gamma^{\prime}$ is $\iota$ and the slope of $\iota^{\prime}\left(r_{j}\right)$ is $\left(a_{1 j}, a_{2 j}, \ldots, a_{n j}\right)$. Then $\Gamma^{\prime}$ and $\iota^{\prime}$ give a zero-tension curve. By our assumption that $\Gamma$ is ordinary, we know that $\iota^{\prime}(\Gamma)$ is not contained in any hyperplane. So, by Lemma 5.1, the slopes of the $\iota^{\prime}\left(r_{j}\right)$ span $\mathbb{Q}^{n}$. Since the slopes of the $\iota^{\prime}\left(r_{j}\right)$ are the columns of $A$, this is the same as saying that $A$ has rank $n$.

So we deduce that $A$ has full rank over $\mathbb{Q}$ and therefore we can choose $u_{1}, \ldots, u_{r}$ such that $\prod_{z \in Y_{i}^{+}} z / \prod_{z \in Y_{i}^{-}} z=\prod_{j=1}^{r} u_{j}^{a_{i j}}$ for $i=1, \ldots, n$. This in turn allows us to construct the desired curve $C$ and desired map $\phi$.

We conclude with an example of this construction.

Example 7.6. Consider the zero-tension curve of genus one shown in Figure 3, where the map $\iota$ is simply an injection. We take all of the edges of the square to have length 1 . Then the universal cover, $\tilde{\Gamma}$, is as shown in Figure 4 . We need to pick $q$ with $v(q)=4$; we make the simple choice $q=t^{4}$. We now cut the circuit of $\Gamma$ at the edge labeled $e$ to produce the tree $T$. Set $u_{0}=\operatorname{Span}_{\mathscr{O}}((1,0),(0,1))$ and $u_{1}=$

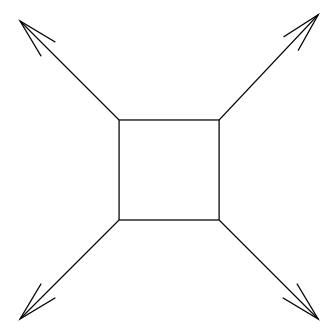

Figure 3. An example of a curve of genus one. 


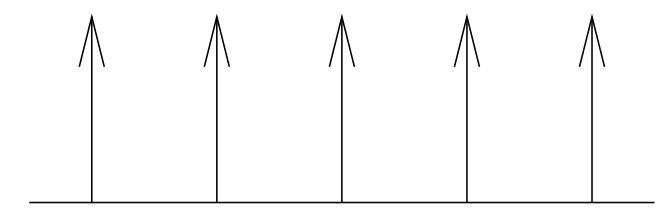

Figure 4. The universal cover of our example.

$\operatorname{Span}_{\mathscr{O}}\left((1,0),\left(0, t^{4}\right)\right)$. We take $U=\left\{u_{0}, t^{0}, t^{1}, t^{2}, t^{3}, u_{1}\right\}$. The reader is invited to check that, indeed, $[U]$ is isometric to $T$ and $\left[\bigcup_{j=-\infty}^{\infty} q^{j}\left(U \backslash\left\{u_{0}, u_{1}\right\}\right)\right]=\left[\left\{t^{k}\right\}_{k \in \mathbb{Z}}\right]$ is isometric to $\tilde{\Gamma}$. The points $t^{0}, t^{1}, t^{2}$ and $t^{3}$ of $\mathbb{P}^{1}(\mathbb{K})$ correspond to the rays of $\Gamma$ with slopes $(1,1),(1,-1),(-1,-1)$ and $(-1,1)$, respectively.

We now need to choose the multisets $Z_{1}^{+}, Z_{1}^{-}, Z_{2}^{+}$and $Z_{2}^{-}$. First we pick subsets $Y_{i}^{ \pm}$of $U$ :

$$
\begin{array}{ll}
Y_{1}^{+}=\left\{t^{0}, t^{1}\right\}, & Y_{1}^{-}=\left\{t^{2}, t^{3}\right\}, \\
Y_{2}^{+}=\left\{t^{0}, t^{3}\right\}, & Y_{2}^{-}=\left\{t^{1}, t^{2}\right\} .
\end{array}
$$

We then modify the $Y$ 's to produce the $Y$ 's. Specifically, we must multiply one of the members of $Y_{1}^{+}$by $q$. We obtain

$$
Y_{1}^{+}=\left\{t^{4}, t^{1}\right\}, \quad Y_{1}^{-}=\left\{t^{2}, t^{3}\right\}, \quad Y_{2}^{+}=\left\{t^{0}, t^{3}\right\}, \quad Y_{2}^{-}=\left\{t^{1}, t^{2}\right\} .
$$

This is the stage in the process where we would perturb the $Y$ 's to produce the $Z$ 's. However, we got lucky this time - we already have $\prod_{z \in Y_{i}^{+}} z=\prod_{z \in Y_{i}^{-}} z$ for $i=1,2$, so no perturbation is necessary and we just take $Z_{i}^{ \pm}=Y_{i}^{ \pm}$. So we take the curve $C$ to be $\mathbb{K}^{*} / t^{4}$ and we take the map $\phi$ to be given by $u \mapsto\left(\phi\left(u ; Z_{1}^{+}, Z_{1}^{-}\right), \phi\left(u ; Z_{2}^{+}, Z_{2}^{-}\right)\right)$. To be completely explicit,

$$
\begin{aligned}
u \mapsto\left(\prod_{j=-\infty}^{0} \frac{\left(1-u / t^{4 j+4}\right)\left(1-u / t^{4 j+1}\right)}{\left(1-u / t^{4 j+2}\right)\left(1-u / t^{4 j+3}\right)} \prod_{j=1}^{\infty} \frac{\left(1-t^{4 j+4} / u\right)\left(1-t^{4 j+1} / u\right)}{\left(1-t^{4 j+2} / u\right)\left(1-t^{4 j+3} / u\right)},\right. \\
\left.\quad \prod_{j=-\infty}^{0} \frac{\left(1-u / t^{4 j}\right)\left(1-u / t^{4 j+3}\right)}{\left(1-u / t^{4 j+1}\right)\left(1-u / t^{4 j+2}\right)} \prod_{j=1}^{\infty} \frac{\left(1-t^{4 j} / u\right)\left(1-t^{4 j+3} / u\right)}{\left(1-t^{4 j+1} / u\right)\left(1-t^{4 j+2} / u\right)}\right) .
\end{aligned}
$$

At this point, the reader may reasonably wonder how to extract an actual equation for the curve $C$. This is basically a problem of implicitization, the recovery of the equation of an algebraic variety from a parametric representation, but it is worse than the usual implicitization problem because the parameterization is analytic, not algebraic. This problem deserves study, which has been begun in [Sturmfels and Yu 2008; Sturmfels et al. 2007]. We will describe here a straightforward but unwieldy method. Let $\Sigma$ denote the complete fan whose rays point in directions $(1,1),(1,-1),(-1,-1)$ and $(-1,1)$. Then, by Proposition 1.2 , the closure of 
$\phi(C)$ in $^{11} \mathrm{X}(\Sigma)$ is torically transverse and has cohomology class corresponding to the linear relation $(1,1)+(1,-1)+(-1,-1)+(-1,1)=0$ between the rays of $\Sigma$. Thus, $\phi(C)$ obeys an equation of the form $P x+Q y+R+S x^{-1}+T y^{-1}=0$, for some $P, Q, R, S$ and $T$ in $\mathbb{K}$. Here we have written the coordinates on $\left(\mathbb{K}^{*}\right)^{2}$ as $x$ and $y$. In order to find $P, Q, R, S$ and $T$, expand the infinite products for $x$ and $y$ as Laurent series around $u-1$, and compare the coefficients of $(u-1)^{k}$ for $k=-1$, $0,1,2,3$. Note that this will express $P, Q, R, S$ and $T$ in terms of infinite sums. There is no reason to expect a better result; our algorithm will usually produce curves defined by equations whose coefficients are not algebraic functions of $t$.

\section{Superabundant curves}

In this section, we prove Theorem 3.4, which we restate for the reader's convenience. Theorem 3.4. Suppose that $\kappa$ has characteristic zero.

Let $(\iota, \Gamma, m)$ be a zero-tension curve of genus one and degree $\delta$. Suppose also that $(\iota, \Gamma, m)$ is well spaced. Then there is a (punctured) genus-one curve $C$ over $\mathbb{K}$ and a map $\phi: C \rightarrow \mathbb{T}(\mathbb{K}, N)$ of degree $\delta$ such that $\iota(\Gamma)=\operatorname{Trop} \phi(C)$.

See the end of Section 3 for the definition of "well spaced". We may as well assume that $\iota(\Gamma)$ is not contained in any hyperplane, as otherwise the problem reduces to constructing a map to a lower-dimensional torus.

For each nonnegative rational number $R$, let $\Delta(R)$ be the subgraph of $\Gamma$ consisting of those points with distance less than or equal to $R$ from the circuit of $\Gamma$. Let $H(R)$ be the affine linear space spanned by $\Delta(R)$. Then $H(R)$ is an increasing chain of affine spaces which increases at a finite number of discrete indices. Let $n_{0}, n_{1}, \ldots, n_{s}=n$ be the dimensions of the various $H_{R}$ 's. (The last term is $n$, because we are assuming that $\iota(\Gamma)$ is not contained in a hyperplane.) The reader may find this section easier to follow under the simplifying assumption that $n_{i}=n_{0}+i$.

Let $m=n_{j}$ for some $j$. Let $R_{m}=\max \{R: \operatorname{dim} H(R) \leq m\}$; let $\Delta_{m}=\Delta\left(R_{m}\right)$ and $H_{m}=H\left(R_{m}\right)$. We will also occasionally need to refer to the open subset of $\Delta_{m}$ consisting of those points at distance strictly less than $R_{m}$ from the circuit of $\Gamma$; we denote this by $\Delta_{m}^{-}$. We can make a change of basis in $N$ such that $H_{m}$ is $\operatorname{Span}_{\mathbb{Q}}\left(e_{1}, e_{2}, \ldots, e_{m}\right)$.

By the arguments in the previous section, we can find $Z_{1}^{ \pm}, \ldots, Z_{n}^{ \pm}$, multisubsets of $\mathbb{P}^{1}(\mathbb{K})$, and $q \in \mathbb{K}^{*}$, such that $f\left(\exists\left(q, Z_{\bullet}^{ \pm}\right)\right)=\iota(\Gamma)$. We can use the perturbation arguments of the preceding section to arrange that $\prod_{z \in Z_{i}^{+}} z=\prod_{z \in Z_{i}^{-}}$for $i=$ $1, \ldots, n_{0}$. However, for $i>n_{0}$, all we can conclude is that

$$
v\left(\prod_{z \in Z_{i}^{+}} z\right)=v\left(\prod_{z \in Z_{i}^{-}} z\right)
$$

${ }^{11}$ Recall that $\mathrm{X}(\Sigma)$ is the toric variety associated to the fan $\Sigma$. 
The strategy of our proof will be to show, by induction on $j$, that we can arrange for the equality $\prod_{z \in Z_{i}^{+}} z=\prod_{z \in Z_{i}^{-}}$to hold for $i \leq n_{j}$.

The case where $j=0$ and $R_{0}=0$ needs to be handled slightly separately; so we will provide the necessary modifications of the argument in that case in square brackets, and address the rest of the proof to the case that $R_{j}>0$.

So, suppose that we have $Z_{1}^{ \pm}, \ldots, Z_{n}^{ \pm}$and $q$ such that $f\left(\beth\left(q, Z_{\bullet}^{ \pm}\right)\right)=\iota(\Gamma)$ and such that $\prod_{z \in Z_{i}^{+}} z=\prod_{z \in Z_{i}^{-}} z$ holds for $i \leq n_{j}$. From now on, we fix $j$; we set $m=n_{j}$ and $m^{\prime}=n_{j+1}$. We introduce the notation $U$ for the group of units $u$ of 0 such that $v(u-1) \geq R_{m}$. [When $j=0$ and $R_{0}=0$, take $U$ to be the group of all units of $\mathrm{O}$; that is, $v^{-1}(0)$.]

Lemma 8.1. The abelian group $U$ is divisible.

Proof. This is where we will use that $\kappa$ has characteristic zero, and hence that $v(k)=0$ for every nonzero integer $j$. Let $u \in U$ and let $j$ be a nonzero integer. Then, since $\mathbb{K}$ is algebraically closed, $u$ has a $j$-th root in $\mathbb{K}$, and even has a root which lies in 0 and reduces to 1 in $\kappa$. (Proof - let $u_{1}, \ldots, u_{j}$ be the roots of $z^{j}-u$ in $\mathbb{K}$. Since $j v\left(u_{i}\right)=v\left(u_{i}^{j}\right)=v(u)=0$, we know that all of the $u_{i}$ are in 0 . Let $\overline{u_{i}}$ be the reduction of $u_{i}$ in $\kappa$. Then we have $z^{j}-1=\prod\left(z-\overline{u_{i}}\right)$ in $\kappa$, so by unique factorization in $\kappa[z]$, one of the $u_{i}$ reduces to 1 in $\kappa$.) So let $(1+\pi)^{j}=u$ with $v(\pi)>0$. Then $u=1+j \pi+\pi^{2} c$ where $c \in \mathcal{O}$. As $v(j)=0$, we have $v(j \pi)<v\left(\pi^{2} c\right)$, so $v(u-1)=v(j \pi)=v(\pi)$ and we deduce that $v(\pi) \geq R_{m}$, so $1+\pi$ is in $U$ as desired.

[When $j=0$ and $R_{0}=0$, we know that any unit of 0 has a $j$-th root in $\mathbb{K}$ since $\mathbb{K}$ is algebraically closed. This $j$-th root must also have valuation zero.]

Lemma 8.2. For each $i$ between 1 and $n$, the ratio $\prod_{z \in Z_{i}^{+}} z / \prod_{z \in Z_{i}^{-}} z$ is in $U$.

Proof. For $i \leq m$, we have $\prod_{z \in Z_{i}^{+}} z / \prod_{z \in Z_{i}^{-}}=1$, which is in $U$. So fix $i>m$. Let $T_{1}, \ldots, T_{r}$ be the components of $\Gamma \backslash \Delta_{m}^{-}$. We note that, for each $k$, we have $\left|Z_{i}^{+} \cap T_{k}\right|=\left|Z_{i}^{-} \cap T_{k}\right|$. This is because, by the zero-tension condition, $\left|Z_{i}^{+} \cap T_{k}\right|-\left|Z_{i}^{-} \cap T_{k}\right|$ is the $i$-th component of the edge of $\Delta_{m}$ pointing inward from $T_{k}$. Since this is an edge of $\Delta_{m}$, that component is zero. So we can pair off the elements of $Z_{i}^{+} \cap T_{K}$ with those of $Z_{i}^{-} \cap T_{k}$. In each pair $\left(z^{+}, z^{-}\right)$, we have $v\left(z^{+} / z^{-}-1\right)=v\left(c\left(z^{+}, \infty ; z^{-}, 0\right)-1\right)$, which is at least $R_{m}$ by Proposition 4.2. Then $\prod_{z \in Z_{i}^{+}} z / \prod_{z \in Z_{i}^{-}} z$ is a product of ratios which are in $U$, and hence is itself in $U$ as we claimed.

[When $j=0$ and $R_{j}=0$, we can't talk about $\Delta_{m}^{-}$. Rather, let $\Delta^{-}$be the interiors of the edges of the loop, so $\Gamma \backslash \Delta^{-}$is a forest with one connected component for each vertex of the loop. Define $T_{1}, T_{2}, \ldots, T_{r}$ to be the connected components of this forest. This time, $\left|Z_{i}^{+} \cap T_{k}\right|-\left|Z_{i}^{-} \cap T_{k}\right|$ is the sum of the $i$-th components of the two edges of the loop incident to $T_{i}$. As before, these edges are in the loop, so 
the $i$-th components are zero. $\left|Z_{i}^{+} \cap T_{k}\right|=\left|Z_{i}^{-} \cap T_{k}\right|$ follows as before, and the rest of the proof does as well.]

We introduce the notation $w_{i}$ for $\prod_{z \in Z_{i}^{+}} z / \prod_{z \in Z_{i}^{-}} z$, which we have just shown to be in $U$. When $1 \leq i \leq m$, then $w_{i}$ is simply 1 .

Let $e_{1}, \ldots, e_{p}$ be the edges of $\Gamma$ which are not in $\Delta_{m}$, but which have endpoints that are in $\Delta_{m}$. Let $s_{1}, \ldots, s_{p}$ be the slopes of these edges, directed away from $\Delta_{m}$. We write the components of $s_{k}$ as $\left(s_{k}^{1}, \ldots, s_{k}^{n}\right)$.

Lemma 8.3. There exist scalars $a_{1}, \ldots, a_{p} \in \mathbb{Q}$ such that $\sum a_{i} s_{i}=0$ and such that if $e_{g}$ and $e_{h}$ are distinct edges with the same endpoint, then $a_{g} \neq a_{h}$.

Proof. Let $t_{i}$ be the image of $s_{i}$ in $\mathbb{Q}^{n} / H_{m}$. We first show that we can find $b_{1}, \ldots, b_{p}$ obeying the required inequalities with $\sum b_{i} t_{i}=0$. Let $L \subset \mathbb{Q}^{p}$ be the space of $p$-tuples of rational numbers $\left(b_{1}, \ldots, b_{p}\right)$ such that $\sum b_{i} t_{i}=0$. We want to show that $L$ has a point which is not in the union of the hyperplanes $b_{g}=b_{h}$, where $(g, h)$ ranges over pairs of indices such that $e_{g}$ and $e_{h}$ share an endpoint. Since $L$ is a linear space, we just need to show that $L$ is not contained in any of these hyperplanes.

Let $e_{g}$ and $e_{h}$ share the endpoint $x$, which is necessarily a boundary vertex of $\Gamma_{m}$. Suppose for the sake of contradiction that $L$ is contained in the hyperplane $a_{g}=a_{h}$. Then in particular, $L$ does not contain any point with $b_{g}=0$ and $b_{h}=1$. This means that $t_{h}$ is not in the span of $\left\{t_{i}\right\}_{i \neq g, h}$. Equivalently, $s_{h}$ is not in $V:=$ $H_{m}+\operatorname{Span}\left(\left\{s_{i}\right\}_{i \neq g, h}\right)$. Let $K$ be a hyperplane in $\mathbb{Q}^{n}$ which contains $V$ and does not contain $s_{h}$. Then $x$ is at distance $R_{m}$ from the loop of $\Gamma$, while every other boundary vertex of $\Gamma \cap \iota^{-1}(K)$ is farther away, contradicting our hypothesis that $\Gamma$ is well spaced. This contradiction shows that we can find the required $b_{i}$.

So, now we have $\left(b_{1}, \ldots, b_{p}\right)$ obeying the required inequalities with $\sum b_{i} s_{i}$ in $H_{m}$. Let $x_{1}, \ldots, x_{r}$ be the boundary vertices of $\Delta_{m}$ and let $u_{k}$ be the slope of the edge of $\Delta_{m}$ pointing inward from $x_{k}$. Then, by Lemma 5.1, the $u_{k}$ span $H_{m}$. Let $\sum b_{i} s_{i}=\sum c_{k} u_{k}$. Now, by the zero-tension condition, $u_{k}=-\sum_{e_{i} \ni x_{k}} s_{i}$. So we have $\sum_{i=1}^{p} b_{i} s_{i}+\sum_{k=1}^{r} c_{k} \sum_{x_{k} \ni e_{i}} s_{i}=0$. We regroup this expression and take the coefficient of $s_{i}$ to be our $a_{i}$. If $e_{g}$ and $e_{h}$ share the endpoint $x_{k}$, then the coefficients of $s_{g}$ and $s_{h}$ in this expression are $b_{g}+c_{k}$ and $b_{h}+c_{k}$. Since $b_{g} \neq b_{h}$, we also have $b_{g}+c_{k} \neq b_{h}+c_{k}$ and we have achieved the goal.

Our strategy will be to choose $u_{1}, \ldots, u_{p} \in U$ and, for each $Z_{i}^{ \pm}$, multiply those elements of $Z_{i}^{ \pm}$which lie in the component of $\Gamma \backslash \Delta_{m}$ containing $e_{k}$ by $u_{k}$. This will have the effect of multiplying $w_{i}$ by

$$
v_{i}:=\prod_{k=1}^{p} u_{k}^{s_{k}^{i}} .
$$


We need to achieve two things: make the new values of $w_{i}$ be 1 for $i \leq m^{\prime}$, and preserve the condition $f(\Xi)=\iota(\Gamma)$.

Now, by Lemma 5.1 applied to $\Delta\left(R_{m}+\epsilon\right)$ for some small $\epsilon$, we know that the $s_{j}$ span $H\left(R_{m^{\prime}}\right)$. Since $U$ is a divisible group, this means that we can arrange to make $v_{i}$ (above) assume any value that we want for $1 \leq i \leq m^{\prime}$. In particular, we can make $w_{1}=w_{2}=\cdots=w_{m^{\prime}}=1$. The trouble is that we might no longer have $I \cong \Gamma$. Now, multiplication by an element of $U$ will fix all of the points of $\Delta_{m}$ and will move each component of $\Gamma \backslash \Delta_{m}$ by an isomorphism. Our only fear is that two of these components which are connected to $\Delta_{m}$ at the same vertex, say $x$, will swing so that the edges at which they attach to $x$, say $e_{c}$ and $e_{d}$, overlap for some length.

Let $\left(a_{1}, \ldots, a_{q}\right) \in \mathbb{Q}^{q}$ be the vector found in Lemma 8.3. Assume that we have arranged for $w_{i}$ to be 1 for $i=1,2, \ldots, n_{j+1}$. Now choose some $u \in U$. For each $Z_{i}^{ \pm}$, take those elements of $Z$ which lie in the component of $\Gamma \backslash \Delta_{m}$ containing $e_{k}$ and multiply them by $u^{a_{k}}$. This will multiply $w_{i}$ by $u^{E}$ where $E=\sum a_{k} s_{k}^{i}=0$; in other words, it will not change $w_{i}$. So this will not break our achievement of making the $w_{i}=1$ (for $i=1, \ldots, m^{\prime}$.) Also, if $e_{c}$ and $e_{d}$ share an end point $x$, then the components of $\Gamma \backslash \Delta_{m}$ containing $e_{c}$ and $e_{d}$ will be multiplied by $u^{a_{c}}$ and $u^{a_{d}}$ respectively, two different scalars, and so, for generic $u \in U$, they will not overlap.

We have now completed the inductive step, showing how to make $w_{i}$ equal to 1 for $i \leq n_{j+1}$. Continuing in this manner, we will eventually have all the $w_{i}$ equal to 1 , and thus the map $\phi$ will be well defined. At that point, we will have a curve $C$ and a map $\phi$ with $\operatorname{Trop} \phi(C)=\iota(\Gamma)$ as desired.

\section{The necessity of well-spacedness and the $j$-invariant}

We have been studying genus-one curves over $C$ by identifying them with Tate curves. It is therefore natural to ask to what extent we may assume that a genus-one curve over $\mathbb{K}$ is a Tate curve.

Theorem 9.1. Let $C$ be a genus-one curve over $\mathbb{K}$ and $\bar{C}$ its projective completion. Let $j$ be the $j$-invariant of $C$. Let $\mathscr{C}$ be the extension of $(\bar{C}, \bar{C} \backslash C)$ to a flat family of stable curves with marked points over $\mathcal{O}$. Then the following are equivalent:

(1) $C$ can be expressed as a Tate curve for some $q \in \mathbb{K}^{*}$ with $v(q)>0$.

(2) $v(j)<0$.

(3) The fiber of $\mathscr{C}$ over Spec $\kappa$ is a union of genus-zero curves.

Moreover, if these equivalent conditions hold, we have $v(q)=-v(j)$.

Proof. The equivalence of (1) and (2) is [Roquette 1970, Section 3, Statement VIIIa]. (Observe that Roquette's conditions (i) and (iii) are automatic when $\mathbb{K}$ is algebraically closed, the latter because the Hasse invariant lives in the group 
$\mathbb{K}^{*} /\left(\mathbb{K}^{*}\right)^{2}$, which is trivial for $\mathbb{K}$ algebraically closed.) If $N$ is the number of points in $\bar{C} \backslash C$, then $\mathscr{C}$ gives a map from Spec(O) to the moduli space of stable genus-one curves with $N$ marked points. Forgetting all but one of the points, we get a map to the moduli space of stable genus-one curves with one marked point. This moduli space is a copy of the projective line, commonly known as the $j$-line. Condition (3) is equivalent to saying that this map sends $\operatorname{Spec} \kappa$ to the point at infinity on the $j$-line, which is exactly what condition (2) says. We have $v(j)=-v(q)$ because $j=q^{-1}+\sum_{i=0}^{\infty} c_{i} q^{i}$, where each $c_{i}$ is an integer and hence has nonnegative valuation.

Combining the above with the second paragraph of Theorem 3.1, we see that if $(\phi, C)$ is trivalent and Trop $\phi(C)$ has first Betti number one, then $C$ is a Tate curve. In this case, we can show that the condition that Trop $\phi(C)$ be well spaced is necessary, and that the length of the loop of Trop $\phi(C)$ is necessarily $-v(j)$. Recall that every nonzero meromorphic function $\phi$ on a Tate curve is of the form $a \prod_{i=1}^{m}\left(\Theta\left(u / z_{i}^{+}\right) / \Theta\left(u / z_{i}^{-}\right)\right)$for some $Z^{+}=\left\{z_{1}^{+}, \ldots, z_{m}^{+}\right\}$and $Z^{-}=\left\{z_{1}^{-}, \ldots, z_{m}^{-}\right\} \subset \mathbb{K}^{*}$ with $\prod z_{i}^{+}=\prod z_{i}^{-}$.

Proposition 9.2. Let $(\phi, C)$ be a trivalent tropical curve of genus one and $(\iota, \Gamma, m)$ a zero-tension curve parameterizing Trop $\phi(C)$, with $b_{1}(\Gamma)=1$ and $\iota$ injective on $\Gamma$. Then the length of the loop of $\Gamma$ is $-v(j(C))$, where $j(C)$ is the $j$-invariant of $C$. Now suppose that $\kappa$ has characteristic zero. Then $(\iota, \Gamma, m)$ is well spaced.

The idea that the length of the circuit of $\Gamma$ is the tropical analogue of the $j$ invariant was suggested in [Mikhalkin 2006] and pursued in [Kerber and Markwig 2009]. E. Katz and H. and T. Markwig have proven that the length of the circuit of $\Gamma$ is the $j$-invariant of a trivalent curve for tropicalizations of cubic curves in $\mathbb{P}^{2}$.

Proof. Our hypotheses are enough to ensure that $C$ is a Tate curve $\mathbb{K}^{*} / q^{\mathbb{Z}}$ for some $q$ and that the map $\phi: C \rightarrow\left(\mathbb{K}^{*}\right)^{n}$ is given by $u \mapsto\left(\phi\left(u ; Z_{1}^{+}, Z_{1}^{-}\right), \ldots, \phi\left(u ; Z_{n}^{+}, Z_{n}^{-}\right)\right)$ for some $Z_{1}^{ \pm}, \ldots, Z_{n}^{ \pm}$.

By our combinatorial construction of $I\left(q, Z_{\bullet}^{ \pm}\right)$, the length of the loop of $\beth$ is $v(q)=-v(j(C))$. Lemma 5.2 ensures that $f$ is injective, and thus the loop of $]$ and the loop of $\Gamma$ have the same length.

Now, assume that $\kappa$ has characteristic zero and (for the sake of contradiction) that $(\iota, \Gamma, m)$ is not well spaced. After a change of coordinates, we may assume that $H$ is the $n$-th coordinate hyperplane. Assume for the sake of contradiction that $x$ is the boundary vertex of $\Gamma \cap H$ which is closest to the loop of $\Gamma$, and let $R$ denote the distance from $x$ to the loop of $\Gamma$. Then $x$ is trivalent by hypothesis. One of the edges of $x$ is between $x$ and the loop of $\Gamma$; this edge is contained in $H$, so its $n$-th component is 0 . Let the $n$-th components of the other two edges be $a$ and $-a$ for some positive integer $a$. Let $\left\{z_{1}^{+}, \ldots, z_{a}^{+}\right\}$and $\left\{z_{1}^{-}, \ldots, z_{a}^{-}\right\}$be the elements of $Z_{n}^{+}$ and $Z_{n}^{-}$respectively which are beyond these edges. Then we have $v\left(z_{i}^{+} / z_{j}^{+}-1\right)>R$ 
for any indices $i$ and $j$ from 1 to $a$ and similarly $v\left(z_{i}^{-} / z_{j}^{-}-1\right)>R$. On the other hand, $v\left(z_{i}^{+} / z_{j}^{-}-1\right)=R$. So

$$
\prod_{i=1}^{a}\left(z_{i}^{+} / z_{i}^{-}\right)=\left(z_{1}^{+} / z_{1}^{-}\right)^{a}\left(1+t^{R} c\right)
$$

for some $c$ with $v(c)>0$ and thus $v\left(\prod_{i=1}^{a}\left(z_{i}^{+} / z_{i}^{-}\right)-1\right)=R$. We have used that $\kappa$ has characteristic zero to deduce that if $v(u-1)>0$, then $v\left(u^{a}-1\right)=v(u-1)$.

Pair off the elements of $Z_{n}^{+} \backslash\left\{z_{1}^{+}, \ldots, z_{a}^{+}\right\}$with elements of $Z_{n}^{-} \backslash\left\{z_{1}^{-}, \ldots, z_{a}^{-}\right\}$ that lie in the same component of $\Gamma \backslash(H \cap \Gamma)$; this is possible by the same argument as in the proof of Lemma 8.2. We write the pairs as $\left(z_{i}^{+}, z_{i}^{-}\right)$for $i>a$. Then in each pair $\left(z^{+}, z^{-}\right)$, we have $v\left(z^{+} / z^{-}-1\right)>R$. But we are supposed to have $\prod_{z \in Z_{n}^{+}} z / \prod_{z \in Z_{n}^{-}} z=1$, so $\prod_{i=1}^{a}\left(z_{i}^{+} / z_{i}^{-}\right)=\left(\prod_{i>a}\left(z_{i}^{+} / z_{i}^{-}\right)\right)^{-1}$. Then the left-hand side differs from 1 by an element of valuation $R$ while the right-hand side differs from 1 by an element of valuation greater than $R$, a contradiction.

\section{Acknowledgements}

This paper, which has been several years in preparation, stems from portions of my Ph.D. dissertation. I am extremely grateful to my advisor Bernd Sturmfels, for his guidance in the writing of the dissertation and afterwards. I want to thank Paul Hacking, Hannah and Thomas Markwig, Grigory Mikhalkin, Sam Payne and Jenia Tevelev for many discussions of my work and theirs, including informing me of many results which were unpublished at the time. Finally, I was aided by several extremely careful referees. During much of the writing of this paper, I have been supported by a research fellowship from the Clay Mathematics Institute.

\section{References}

[Baker and Norine 2007] M. Baker and S. Norine, "Riemann-Roch and Abel-Jacobi theory on a finite graph”, Adv. Math. 215:2 (2007), 766-788. MR 2008m:05167 Zbl 1124.05049

[Baker et al. 2012] M. Baker, S. Payne, and J. Rabinoff, "Nonarchimedean geometry, tropicalization, and metrics on curves", preprint, 2012. arXiv 1104.0320

[Bieri and Groves 1984] R. Bieri and J. R. J. Groves, "The geometry of the set of characters induced by valuations", J. Reine Angew. Math. 347 (1984), 168-195. MR 86c:14001 Zbl 0526.13003

[Bogart et al. 2007] T. Bogart, A. N. Jensen, D. Speyer, B. Sturmfels, and R. R. Thomas, "Computing tropical varieties”, J. Symbolic Comput. 42:1-2 (2007), 54-73. MR 2007j:14103 Zbl 1121.14051

[Draisma 2008] J. Draisma, "A tropical approach to secant dimensions", J. Pure Appl. Algebra 212:2 (2008), 349-363. MR 2008j:14102 Zbl 1126.14059

[Einsiedler et al. 2006] M. Einsiedler, M. Kapranov, and D. Lind, "Non-Archimedean amoebas and tropical varieties", J. Reine Angew. Math. 601 (2006), 139-157. MR 2007k:14038 Zbl 1115.14051

[Fulton 1993] W. Fulton, Introduction to toric varieties, Annals of Mathematics Studies 131, Princeton University Press, 1993. MR 94g:14028 Zbl 0813.14039 
[Fulton and Sturmfels 1997] W. Fulton and B. Sturmfels, "Intersection theory on toric varieties", Topology 36:2 (1997), 335-353. MR 97h:14070 Zbl 0885.14025

[Gathmann and Kerber 2008] A. Gathmann and M. Kerber, "A Riemann-Roch theorem in tropical geometry", Math. Z. 259:1 (2008), 217-230. MR 2009a:14014 Zbl 1187.14066

[Gathmann et al. 2009] A. Gathmann, M. Kerber, and H. Markwig, "Tropical fans and the moduli spaces of tropical curves", Compos. Math. 145:1 (2009), 173-195. MR 2009m:14085 Zbl 1169. 51021

[Helm and Katz 2012] D. Helm and E. Katz, "Monodromy filtrations and the topology of tropical varieties”, Canad. J. Math. 64:4 (2012), 845-868. MR 2957233 Zbl 06064256

[Katz 2012] E. Katz, "Lifting tropical curves in space and linear systems on graphs", Adv. Math. 230:3 (2012), 853-875. MR 2921163 Zbl 06059012

[Kerber and Markwig 2009] M. Kerber and H. Markwig, "Counting tropical elliptic plane curves with fixed j-invariant”, Comment. Math. Helv. 84:2 (2009), 387-427. MR 2010c:14079 Zbl 1205.14071

[Mikhalkin 2005] G. Mikhalkin, "Enumerative tropical algebraic geometry in $\mathbb{R}^{2}$ ", J. Amer. Math. Soc. 18:2 (2005), 313-377. MR 2006b:14097 Zbl 1092.14068

[Mikhalkin 2006] G. Mikhalkin, “Tropical geometry and its applications”, pp. 827-852 in International Congress of Mathematicians (Madrid, 2006), vol. 2, edited by M. Sanz-Solé et al., Eur. Math. Soc., Zürich, 2006. MR 2008c:14077 Zbl 1103.14034 arXiv math/0601041

[Mikhalkin 2007] G. Mikhalkin, "Moduli spaces of rational tropical curves", pp. 39-51 in Proceedings of Gökova Geometry-Topology Conference 2006 (Gökova, 2006), edited by S. Akbulut et al., International Press, Somerville, MA, 2007. MR 2009i:14014 Zbl 1203.14027 arXiv 0704.0839

[Morgan and Shalen 1984] J. W. Morgan and P. B. Shalen, "Valuations, trees, and degenerations of hyperbolic structures, I”, Ann. of Math. (2) 120:3 (1984), 401-476. MR 86f:57011 Zbl 0583.57005

[Nishinou 2010] T. Nishinou, "Correspondence theorems for tropical curves", preprint, 2010. arXiv 0912.5090

[Nishinou and Siebert 2006] T. Nishinou and B. Siebert, "Toric degenerations of toric varieties and tropical curves", Duke Math. J. 135:1 (2006), 1-51. MR 2007h:14083 Zbl 1105.14073 arXiv math/0409060

[Payne 2009a] S. Payne, "Analytification is the limit of all tropicalizations", Math. Res. Lett. 16:3 (2009), 543-556. MR 2010j:14104 Zbl 1193.14077

[Payne 2009b] S. Payne, "Fibers of tropicalization", Math. Z. 262:2 (2009), 301-311. MR 2010c: 14081 Zbl 1222.14130

[Roquette 1970] P. Roquette, Analytic theory of elliptic functions over local fields, Hamburger Mathematische Einzelschriften (N.F.) 1, Vandenhoeck and Ruprecht, Göttingen, 1970. MR 41 \#5376 Zbl 0194.52002

[Shustin and Tyomkin 2006] E. Shustin and I. Tyomkin, "Patchworking singular algebraic curves, I", Israel J. Math. 151 (2006), 125-144. MR 2007b:14056 Zbl 1128.14019

[Speyer and Sturmfels 2004] D. Speyer and B. Sturmfels, "The tropical Grassmannian", Adv. Geom. 4:3 (2004), 389-411. MR 2005d:14089 Zbl 1065.14071

[Sturmfels 1996] B. Sturmfels, Gröbner bases and convex polytopes, University Lecture Series 8, American Mathematical Society, Providence, RI, 1996. MR 97b:13034 Zbl 0856.13020

[Sturmfels 2002] B. Sturmfels, Solving systems of polynomial equations, CBMS Regional Conference Series in Mathematics 97, American Mathematical Society, Providence, RI, 2002. MR 2003i:13037 Zbl 1101.13040 
[Sturmfels and Yu 2008] B. Sturmfels and J. Yu, "Tropical implicitization and mixed fiber polytopes", pp. 111-131 in Software for algebraic geometry (Minneapolis, MN, 2006), edited by M. E. Stillman et al., IMA Vol. Math. Appl. 148, Springer, New York, 2008. MR 2009m:14089 Zbl 1143.14313

[Sturmfels et al. 2007] B. Sturmfels, J. Tevelev, and J. Yu, "The Newton polytope of the implicit equation”, Mosc. Math. J. 7:2 (2007), 327-346. MR 2008f:14073 Zbl 1133.13026 arXiv math/0607368

Communicated by Bernd Sturmfels

Received 2013-06-12 Revised 2013-12-20 Accepted 2014-01-29

speyer@umich.edu

Department of Mathematics, University of Michigan, 2844 East Hall, 530 Church Street,

Ann Arbor, MI 48109-1043, United States 


\section{Algebra \& Number Theory}

msp.org/ant

\section{EDITORS}

MANAGING EDITOR

Bjorn Poonen

Massachusetts Institute of Technology

Cambridge, USA

\author{
EDITORIAL BOARD CHAIR \\ David Eisenbud \\ University of California \\ Berkeley, USA
}

BOARD OF EDITORS

Georgia Benkart

Dave Benson

Richard E. Borcherds

John H. Coates

J-L. Colliot-Thélène

Brian D. Conrad

Hélène Esnault

Hubert Flenner

Edward Frenkel

Andrew Granville

Joseph Gubeladze

Roger Heath-Brown

Craig Huneke

Yujiro Kawamata

János Kollár

Yuri Manin

Barry Mazur

Philippe Michel

Susan Montgomery
University of Wisconsin, Madison, USA

University of Aberdeen, Scotland

University of California, Berkeley, USA

University of Cambridge, UK

CNRS, Université Paris-Sud, France

University of Michigan, USA

Freie Universität Berlin, Germany

Ruhr-Universität, Germany

University of California, Berkeley, USA

Université de Montréal, Canada

San Francisco State University, USA

Oxford University, UK

University of Virginia, USA

University of Tokyo, Japan

Princeton University, USA

Northwestern University, USA

Harvard University, USA

École Polytechnique Fédérale de Lausanne

University of Southern California, USA
Shigefumi Mori

Raman Parimala

Jonathan Pila

Anand Pillay

Victor Reiner

Peter Sarnak

Joseph H. Silverman

Michael Singer

Vasudevan Srinivas

J. Toby Stafford

Bernd Sturmfels

Richard Taylor

Ravi Vakil

Michel van den Bergh

Marie-France Vignéras

Kei-Ichi Watanabe

Efim Zelmanov

Shou-Wu Zhang
RIMS, Kyoto University, Japan

Emory University, USA

University of Oxford, UK

University of Notre Dame, USA

University of Minnesota, USA

Princeton University, USA

Brown University, USA

North Carolina State University, USA

Tata Inst. of Fund. Research, India

University of Michigan, USA

University of California, Berkeley, USA

Harvard University, USA

Stanford University, USA

Hasselt University, Belgium

Université Paris VII, France

Nihon University, Japan

University of California, San Diego, USA

Princeton University, USA

\section{PRODUCTION}

production@msp.org

Silvio Levy, Scientific Editor

See inside back cover or msp.org/ant for submission instructions.

The subscription price for 2014 is US $\$ 225 /$ year for the electronic version, and $\$ 400 /$ year ( $\$ 55$, if shipping outside the US) for print and electronic. Subscriptions, requests for back issues and changes of subscribers address should be sent to MSP.

Algebra \& Number Theory (ISSN 1944-7833 electronic, 1937-0652 printed) at Mathematical Sciences Publishers, 798 Evans Hall \#3840, c/o University of California, Berkeley, CA 94720-3840 is published continuously online. Periodical rate postage paid at Berkeley, CA 94704, and additional mailing offices.

ANT peer review and production are managed by EditFLOw ${ }^{\circledR}$ from Mathematical Sciences Publishers.

\section{PUBLISHED BY}

mathematical sciences publishers

nonprofit scientific publishing

http://msp.org/

(C) 2014 Mathematical Sciences Publishers 


\section{Algebra \& Number Theory}

Volume $8 \quad$ No. $4 \quad 2014$

The derived moduli space of stable sheaves

Kai Behrend, Ionut Ciocan-Fontanine, Junho Hwang and Michael Rose

Averages of the number of points on elliptic curves

Greg Martin, Paul Pollack and Ethan Smith

Noncrossed product bounds over Henselian fields

Timo Hanke, DanNy NeFtin and JaCk SONN

Yangians and quantizations of slices in the affine Grassmannian

Joel Kamnitzer, Ben Webster, Alex Weekes and Oded Yacobi

Equidistribution of values of linear forms on quadratic surfaces

OLIVER SARGENT

Posets, tensor products and Schur positivity

Vyjayanthi Chari, Ghislain Fourier and Daisuke SAGaKi

Parameterizing tropical curves I: Curves of genus zero and one

DAVID E. SPEYER

Pair correlation of angles between reciprocal geodesics on the modular surface

Florin P. Boca, Vicențiu Pașol, AleXandru A. Popa and AleXandru

ZAHARESCU

Étale contractible varieties in positive characteristic

ARMin HolschbaCh, JohanNes SCHMIDT and JaKob STIX 